

\section{Handbagk of FOOD ANALYSIS INSTRUMENTS}





\section{Handbogk of}

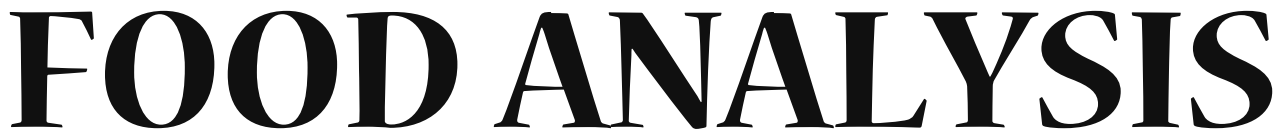

INSTRUMENTS

\section{Edited by}

Semih 0̈tleş

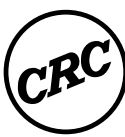

CRC Press

Taylor \& Francis Group

Boca Raton London New York

CRC Press is an imprint of the

Taylor \& Francis Group, an informa business 


\section{CRC Press}

Taylor \& Francis Group

6000 Broken Sound Parkway NW, Suite 300

Boca Raton, FL 33487-2742

(C) 2009 by Taylor \& Francis Group, LLC

CRC Press is an imprint of Taylor \& Francis Group, an Informa business

No claim to original U.S. Government works

Printed in the United States of America on acid-free paper

10987654321

International Standard Book Number-13: 978-1-4200-4566-6 (Hardcover)

This book contains information obtained from authentic and highly regarded sources. Reasonable efforts have been made to publish reliable data and information, but the author and publisher cannot assume responsibility for the validity of all materials or the consequences of their use. The authors and publishers have attempted to trace the copyright holders of all material reproduced in this publication and apologize to copyright holders if permission to publish in this form has not been obtained. If any copyright material has not been acknowledged please write and let us know so we may rectify in any future reprint.

Except as permitted under U.S. Copyright Law, no part of this book may be reprinted, reproduced, transmitted, or utilized in any form by any electronic, mechanical, or other means, now known or hereafter invented, including photocopying, microfilming, and recording, or in any information storage or retrieval system, without written permission from the publishers.

For permission to photocopy or use material electronically from this work, please access www.copyright.com (http:// www.copyright.com/) or contact the Copyright Clearance Center, Inc. (CCC), 222 Rosewood Drive, Danvers, MA 01923, 978-750-8400. CCC is a not-for-profit organization that provides licenses and registration for a variety of users. For organizations that have been granted a photocopy license by the CCC, a separate system of payment has been arranged.

Trademark Notice: Product or corporate names may be trademarks or registered trademarks, and are used only for identification and explanation without intent to infringe.

\section{Library of Congress Cataloging-in-Publication Data}

Handbook of food analysis instruments / editor, Semih Otles.

p. cm.

Includes bibliographical references and index.

ISBN 978-1-4200-4566-6 (alk. paper)

1. Food--Analysis--Equipment and supplies--Handbooks, manuals, etc. I. Ötles, Semih. II. Title.

TX541.H365 2008

664'.07--dc22

2008013711

Visit the Taylor \& Francis Web site at

http://www.taylorandfrancis.com

and the CRC Press Web site at

http://www.crcpress.com 


\section{Contents}

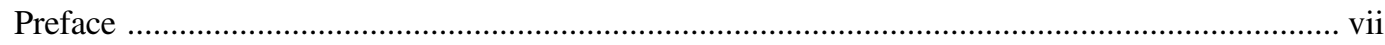

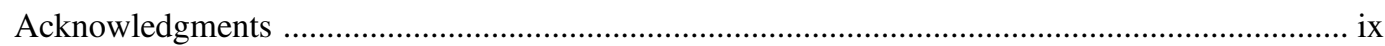

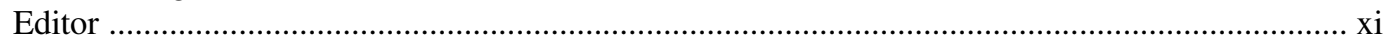

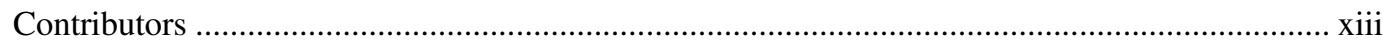

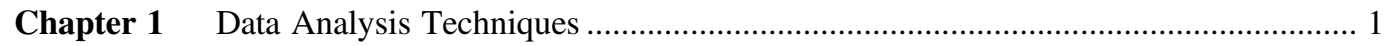

Michael H. Tunick

Chapter 2 Microextraction Methods in Food Analysis

Kathy Ridgway, Sam P.D. Lalljie, and Roger M. Smith

Chapter 3 Supercritical Fluid Extraction in Food Analysis

Ruhan Askin, Motonobu Goto, and Mitsuru Sasaki

Chapter 4 Microwave-Assisted Processes in Food Analysis.... 57

Jacqueline M.R. Bélanger and J.R. Jocelyn Paré

Chapter 5 Ultrasound-Assisted Extraction in Food Analysis

Farid Chemat, Valérie Tomao, and Matthieu Virot

Chapter 6 Advances in High-Performance Liquid Chromatography

and Its Application to the Analysis of Foods and Beverages

Peter Varelis

Chapter 7 Gas Chromatography in Food Analysis

Jana Hajslova and Tomas Cajka

Chapter 8 Preparative Layer Chromatography in Food Analysis

Joseph Sherma

Chapter 9 Ion Chromatography in Food Analysis

William R. LaCourse

Chapter 10 Mass Spectrometry and Hyphenated Instruments in Food Analysis

Tomas Cajka, Jana Hajslova, and Katerina Mastovska 
Chapter 11 Instruments to Analyze Food Colors 229

Carmen Socaciu and Horst A. Diehl

Chapter 12 High-Resolution Near-Infrared and Nuclear Magnetic Resonance Analysis of Food and Grain Composition.

Ion C. Baianu and T. You

Chapter 13 Nuclear Magnetic Resonance Spectroscopy in Food Analysis 281

Francesco Capozzi and Mauro A. Cremonini

Chapter 14 Atomic Absorption, Atomic Emission, and Inductively Coupled Plasma Spectroscopies in Food Analysis

John R. Dean and Renli Ma

Chapter 15 Autofluorescence Spectroscopy in Food Analysis

Charlotte Mфller Andersen, Jens Petter Wold, and Sфren Balling Engelsen

Chapter 16 Electronic Nose Technology in Food Analysis

Figen Korel and Murat Ö. Balaban

Chapter 17 Electroanalytical Techniques and Instrumentation in Food Analysis 379

Rubin Gulaboski and Carlos M. Pereira

Chapter 18 Capillary Electrophoresis in Food Analysis 403

Carmen García-Ruiz and Maria Luisa Marina

Chapter 19 Gel Electrophoresis in Food Analysis 423

Reiner Westermeier and Burghardt Scheibe

Chapter 20 Multiplexed Immunoassays in Food Analysis 439

Chien-Sheng Chen, Antje J. Baeumner, and Richard A. Durst

Chapter 21 Rheological Instruments in Food Analysis 461 Nesli Sozer and Jozef L. Kokini

Chapter 22 Scanning Electron and Transmission Electron Microscopies in Food Analysis 495

José M. Aguilera and Pedro Bouchon

Index 


\section{Preface}

The analysis of foods-identification, speciation, and determination of components, additives, and contaminants in different raw materials and products-is a critical endeavor in food processing and manufacturing companies since the presence and interactions of various compounds in foods during storage and processing have an impact on all aspects of the quality of food products.

The application of proper methods, suitable for analysis of different matrixes with the required method of detection, is crucial for food quality and safety control during production and marketing. In the course of the twenty-first century, analytic methods used in food science have evolved considerably.

While traditional methods are still used, most analysis now involves the use of increasingly sophisticated instruments. Although there are a number of books that explain the principles of food analysis, describe how to conduct food analysis, and discuss test results, there are few books that focus on understanding the actual instruments used in the analysis. Such instruments are used for a wide variety of tasks, including analyzing the degradation of edible oils or the vitamins in baby food; or quantifying food additives, pesticide residues, or the color in packaging materials; or determining the distinct aroma found in natural products. This handbook has been prepared by a team of food scientists/chemists/biochemists who have extensive personal experience in research of food analysis and practical food control in the industry. This handbook aids the analyst by providing a valuable reference regarding the newly developed instruments and methods of analysis of food components and additives.

The handbook, contributed to by 44 leading scientists, many of whom actually developed or refined the techniques and instruments, presents each technique in a uniform format, in a style that can be understood by a reader who is not familiar with the particular technique. Each chapter is structured to provide a description of the information the technique can provide, a simple explanation of how it works, examples of its application, and practical information such as names of instrument vendors, relative costs of instruments and materials, training and education of personnel, and references for more detailed information. This format also facilitates comparison of techniques. The use of different authors to cover a broad spectrum of techniques resulted in some differences of style, but overall the handbook achieved its goal.

The handbook comprises a preface, a contributor list, and a subject index and 22 chapters, which take the reader through brief and accessible descriptions of instruments of analysis of food components and additives. Each chapter in the handbook focuses on a specific type of instrument: capillary electrophoresis, high-performance liquid chromatography (HPLC), nuclear magnetic resonance (NMR), or microwave-assisted process, etc., among many others. Each chapter follows a consistent format, examining the operating principles of a particular technique, its definitions, theory, and applications to food analysis. Each chapter is introduced by an overview written by the chapter authors.

The introductory chapter, "Data Analysis Techniques," covers topics relevant to all techniques, including calibration, standard addition, internal standards, selectivity, accuracy, precision, detection limit, quantification limit, range, robustness, speed, and convenience. The remaining 21 chapters address the major areas of food analysis instruments for sample processing of foods and for food analysis. Chapters 2 and 3 explain sample processing focused on purification and enrichment (Chapter 2: microextraction methods in food analysis such as LPME, SPE, SPME, and SBSE) and extraction while Chapters 3 through 5 explain supercritical fluid extraction, microwave-assisted processes, and ultrasound-assisted extraction. The other chapters explain food analysis instruments based on chromatography (Chapters 6 through 9 and Chapter 18: high-pressure liquid, gas, 
preparative layer, ion, and capillary chromatographies); mass spectroscopy and hyphenated techniques (Chapter 10: MS, GC-MS, HPLC-MS, ICP-MS, etc.); physical parameters such as optical (Chapters 11 through 15: color measurements; near infrared; nuclear magnetic resonance; Raman, atomic absorption, emission, and inductively coupled plasma; and autofluorescence spectroscopies); electrical (Chapter 17: electroanalytical techniques and instrumentation); rheological (Chapter 21: rheological instruments); dedicated systems (Chapter 16: electronic nose technology); and based on biological techniques (Chapters 19 through 22: gel electrophoresis, multiplexed immunoassays, and scanning electron and transmission electron microscopies).

The handbook addresses primarily food science graduate students, food chemists in industry and food quality control, as well as persons who participate in continuing education systems. Many topics will also be of interest to students of chemistry and biology. Some chapters of the handbook could as well be useful to readers interested in the quality of food.

Semih Ötleş 


\section{Acknowledgments}

The editor would like to thank all the contributors for the hard work they put into the various chapters of the handbook. The editor also thanks the people at Taylor \& Francis Group/CRC Press for their help with the production of this book and also expresses his sincere gratitude to Stephen M. Zollo, Taylor \& Francis Group, for his help in preparing the handbook. Finally, special thanks to my wife, Sema Ötleş, for her patience during the preparation and publication steps of the handbook. 



\section{Editor}

Semih Ötleş, a native of Izmir, Turkey, obtained his BSc from the Department of Food Engineering (Ege University, Izmir, Turkey) in 1980. During his assistantship at Ege University in 1985, he received an MS in food chemistry, and in 1989, after completing his thesis research on the instrumental analysis and chemistry of vitamins in foods he received a $\mathrm{PhD}$ in food chemistry from Ege University. During 1991-1992, he completed his postdoctoral training on an Organisation for Economic Co-Operation and Development (OECD) postdoctoral fellowship at the Research Center for Meat Technology, Melle, Ghent University, Belgium. Later, he joined the Department of Food Engineering at Ege University as a scientist in food chemistry, and was promoted to associate professor in 1993 and professor in 2000. During the summer of 2005, he was the visiting professor at Kumamoto University, Kumamoto, Japan, as a fellow of the Japan Society of the Promotion of Science (JSPS). During 1996-1998, he was the deputy director at the Ege Vocational School of Higher Studies. Since 2003, he has been the vice dean of the engineering faculty, Ege University. Also, he is a member of the steering committee in the geriatrics department of the medical faculty, Ege University.

The research activities of Professor Ötles have focused on the instrumental analysis of food compounds. He began a series of projects on separation and analysis techniques using highperformance liquid chromatography (HPLC), first for analysis of vitamins in foods, then proteins, carbohydrates, and most recently carotenoids. Other activities span the fields of SFE (supercritical fluid extraction), GC (gas chromatography), GC/MS (mass spectrometry) analysis, soy chemistry, aromatics, medical and functional foods, and nutraceutical chemistry, including multiresidue analysis of various foods, and $n-3$ fatty acids in fish oils.

Professor Ötleş has authored or coauthored more than 150 publications (technical papers, book chapters, and books) and has presented seminars in these areas. He is a member of several scientific societies, associations, and organizations including the Asian Pacific Organization for Cancer Prevention and International Society of Food Physicists. He is a member of the steering committee of the Food Safety Association, Istanbul, Turkey and the APOCP (Asian Pacific Organization for Cancer Prevention) local scientific bureau, and is a Turkish representative of the International Society of Food Physicists (ISFP), and has organized international congresses on diet/cancer, functional foods, and food physics.

Dr. Ötleş is a member of the editorial advisory boards of the Asian Pacific Journal of Cancer Prevention (APJCP); FSTA (Food Science and Technology Abstracts) of IFIS (International Food Information Service); Current Topics in Nutraceutical Research; Electronic Journal of Environmental, Agricultural and Food Chemistry (EJEAFChe); Newsline (IUFoST, Corr.); Journal of Oil, Soap, Cosmetics; Turkish World Food; Acta Scientiarum Polonorum; Trends in Food Science and Technology; Pakistan Journal of Nutrition; Journal of Food Technology; Turkish Journal of Toxicology; Electronic Journal of Polish Agricultural Universities; CHI; Plastic and Packaging Technology; Genes \& Nutrition; Popular Health Journal; Drink Tech, Advances in Food Sciences; and Keyfood Magazine. He is also a referee/reviewer for The Journal of AOAC International, Journal of Experimental Marine Biology and Ecology, Journal of Medical Foods, die Nahrung, Journal of Alternative and Complementary Medicine, Journal of Harvest, The Analyst, AU Journal of Science and Technology, Turkish Journal of Fisheries and Aquatic Sciences, GOPU Journal of Agriculture Faculty, Journal of Agricultural and Food Chemistry, Electronic Journal of Biotechnology, Industrial \& Engineering Chemistry Research, Journal of Food Processing and Preservation, Cancer Causes and Control, and Food Chemistry. 



\section{Contributors}

José M. Aguilera

Department of Chemical and Bioprocess

Engineering

Universidad Católica de Chile

Santiago, Chile

\section{Charlotte Møller Andersen}

Department of Food Science

University of Copenhagen

Frederiksberg, Denmark

\section{Ruhan Askin}

Department of Applied Chemistry and Biochemistry

Kumamoto University

Kumamoto, Japan

\section{Antje J. Baeumner}

Department of Biological and Environmental

Engineering

Cornell University

Ithaca, New York

\section{Ion C. Baianu}

Food Science and Human Nutrition and Nuclear

Engineering Departments

University of Illinois

Urbana, Illinois

\section{Murat Ö. Balaban}

Fishery Industrial Technology Center

University of Alaska, Fairbanks

Kodiak, Alaska

\section{Jacqueline M.R. Bélanger}

Green Technologies Division

Environment Canada

Ottawa, Ontario, Canada

\section{Pedro Bouchon}

Department of Chemical and Bioprocess

Engineering

Universidad Católica de Chile

Santiago, Chile

\section{Tomas Cajka}

Department of Food Chemistry and Analysis Institute of Chemical Technology

Prague, Czech Republic

\section{Francesco Capozzi}

Department of Food Science

University of Bologna

Cesena, Italy

\section{Farid Chemat}

Sécurité et Qualité des Produits d'Origine

Végétale

University of Avignon

Avignon, France

\section{Chien-Sheng Chen}

Department of Food Science

National Taiwan Ocean University

Keelung, Taiwan

\section{Mauro A. Cremonini}

Department of Food Science

University of Bologna

Cesena, Italy

\section{John R. Dean}

School of Applied Sciences

Northumbria University

Newcastle upon Tyne, United Kingdom

\section{Horst A. Diehl}

Institute of Biophysics

University of Bremen

Bremen, Germany

\section{Richard A. Durst}

Department of Food Science and Technology

Department of Biological and Environmental

Engineering

Cornell University

Ithaca, New York 
Søren Balling Engelsen

Department of Food Science

The Royal Veterinary and Agricultural

University

Frederiksberg, Denmark

\section{Carmen García-Ruiz}

Department of Analytical Chemistry

University of Alcalá

Madrid, Spain

\section{Motonobu Goto}

Department of Applied Chemistry and Biochemistry

Kumamoto University

Kumamoto, Japan

\section{Rubin Gulaboski}

Faculdade de Ciências

Universidade do Porto

Porto, Portugal

\section{Jana Hajslova}

Department of Food Chemistry and Analysis Institute of Chemical Technology

Prague, Czech Republic

\section{Jozef L. Kokini}

Department of Food Science and Center for Advanced Food Technology

Rutgers University

New Brunswick, New Jersey

\section{Figen Korel}

Izmir Institute of Technology

Food Engineering Department

Urla, Izmir, Turkey

\section{William R. LaCourse}

Department of Chemistry and Biochemistry

University of Maryland

Baltimore, Maryland

\section{Sam P.D. Lalljie}

Safety and Environmental Assurance Centre Unilever

Bedfordshire, United Kingdom

\section{Renli Ma}

School of Applied Sciences

Northumbria University

Newcastle upon Tyne, United Kingdom

\section{Maria Luisa Marina}

Department of Analytical Chemistry

University of Alcalá

Madrid, Spain

\section{Katerina Mastovska}

Agricultural Research Service

Eastern Regional Research Center

U.S. Department of Agriculture

Wyndmoor, Pennsylvania

\section{J.R. Jocelyn Paré}

Green Technologies Division

Environment Canada

Ottawa, Ontario, Canada

\section{Carlos M. Pereira}

Departmento de Química

Faculdade de Ciências da Universidade

do Porto

Porto, Portugal

\section{Kathy Ridgway}

Safety and Environmental Assurance Centre

Unilever

Bedfordshire, United Kingdom

Mitsuru Sasaki

Department of Applied Chemistry and Biochemistry

Kumamoto University

Kumamoto, Japan

\section{Burghardt Scheibe}

Protein Sciences

GE-Healthcare Europe

Munich, Germany

\section{Joseph Sherma}

Department of Chemistry

Lafayette College

Easton, Pennsylvania 
Roger M. Smith

Department of Chemistry

Loughborough University

Leicestershire, United Kingdom

\section{Carmen Socaciu}

Department of Chemistry and Biochemistry

University of Agricultural Sciences and Veterinary Medicine

Cluj-Napoca, Romania

\section{Nesli Sozer}

Food Engineering Department

Gaziantep University

Gaziantep, Turkey

\section{Valérie Tomao}

Sécurité et Qualité des Produits d'Origine Végétale

University of Avignon

Avignon, France

\section{Michael H. Tunick}

Dairy Processing and Products Research Unit Eastern Regional Research Center

U.S. Department of Agriculture

Wyndmoor, Pennsylvania

\section{Peter Varelis}

Department of Biological, Chemical, and Physical Sciences

Illinois Institute of Technology

Summit Argo, Illinois

\section{Matthieu Virot}

Sécurité et Qualité des Produits d'Origine

Végétale

University of Avignon

Avignon, France

\section{Reiner Westermeier}

Protein Sciences

GE-Healthcare Europe

Munich, Germany

\section{Jens Petter Wold}

Matforsk

Osloveien, Norway

\section{T. You}

Food Science and Human Nutrition and Nuclear Engineering Departments

University of Illinois

Urbana, Illinois 



\title{
1 Data Analysis Techniques
}

\author{
Michael H. Tunick
}

\section{CONTENTS}

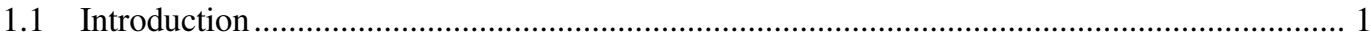

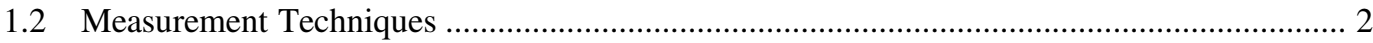

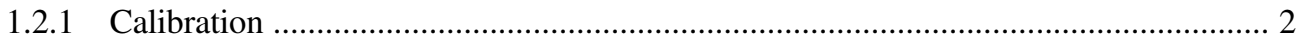

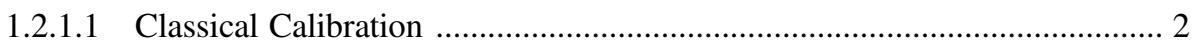

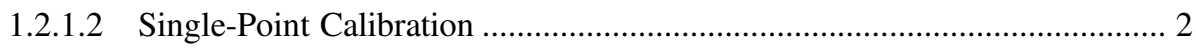

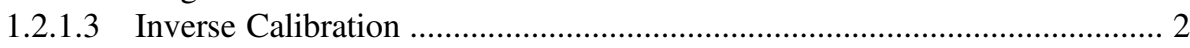

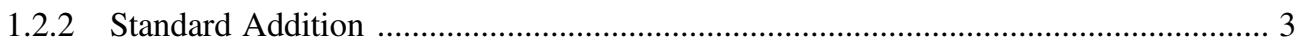

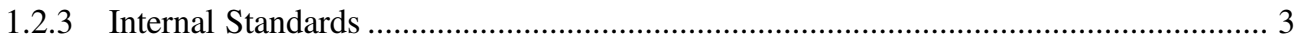

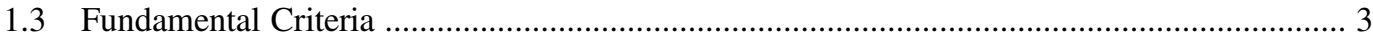

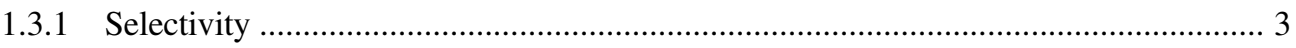

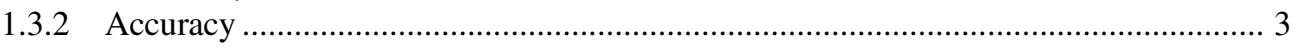

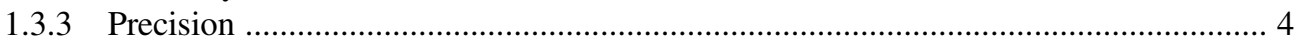

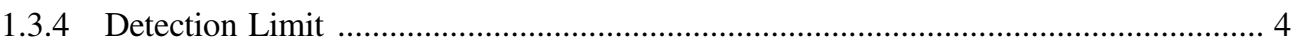

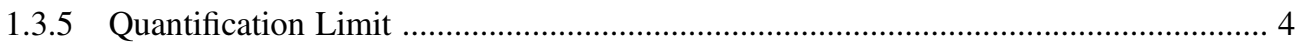

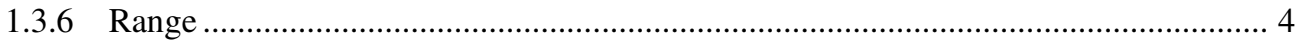

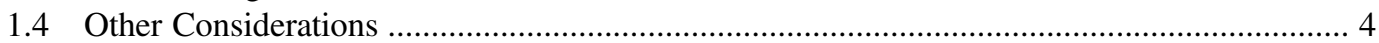

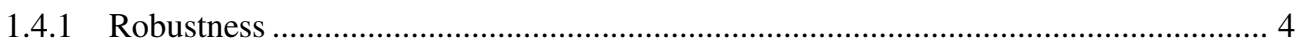

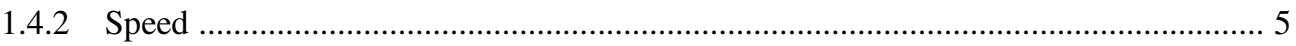

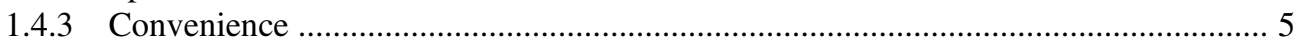

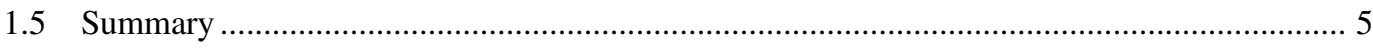

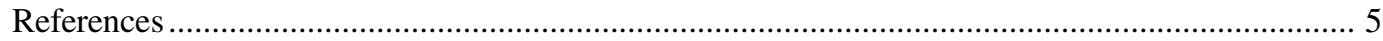

\subsection{INTRODUCTION}

When a food scientist needs to measure a quantity, such as sample mass or volume, he or she performs a direct measurement, having a good idea of the accuracy and precision involved. But when the concentration of a substance in a sample matrix must be found, the analyst has to make an indirect measurement by calculating the quantity from the measurement of other quantities [1]. Indirect measurements are obtained by correlating a result with sample concentration, which introduces the possibility of decreased accuracy and precision. An equation relating analyte concentration and the instrumental response is formed by using standards and calibrations, and then applied to predict the concentration of the unknown [2]. The procedure must demonstrate traceability, defined as an unbroken chain of comparisons from the measurement to the appropriate national or international standards [3]. This chapter outlines the various techniques available for relating the output of an instrument with the quantity being sought and the necessary criteria involved. 


\subsection{MEASUREMENT TECHNIQUES}

\subsubsection{Calibration}

\subsubsection{Classical Calibration}

The common methods for relating concentration and instrumental response are calibration, standard addition, and the use of internal standards. A classical calibration, also called the standard series method or external standards method, is frequently employed in analytical chemistry. A series of samples containing known concentrations of the substance in question are analyzed, and the resulting responses are plotted against concentration to obtain a calibration curve. The curve is often linear, following the equation

$$
y=m c+b
$$

where

$y$ is the instrumental response

$c$ is the analyte concentration

$m$ is the slope (defined as the sensitivity [3])

$b$ is the $y$-intercept, which corresponds to the value for the blank

When a sample containing an unknown $c$ is analyzed, the response is substituted into the equation to obtain the concentration. Linear regression plots and straight linear plots are most often employed, but quadratic regressions, log plots, etc., are sometimes utilized. For example, the author uses a nitrogen analyzer that is calibrated with an ethylenediaminetetraacetic acid (EDTA) standard that contains $9.56 \%$ nitrogen. The EDTA itself was calibrated by the instrument manufacturer against a carbon-hydrogen-nitrogen standard from NIST (National Institute of Standards and Technology), thus establishing traceability. The instrument plots the weights of EDTA against the areas of the response, and calculates fixed and regressed linear, quadratic, and cubic calibration curves. The simplest curve yielding with an acceptable correlation coefficient ( 0.999 or higher) is selected for the calibration curve.

Mitchell et al. [4] detailed a rigorous method for performing this type of calibration. After selecting and analyzing the standards, the regression order is selected, outliers are rejected, and regression equations are obtained with confidence bands.

\subsubsection{Single-Point Calibration}

When a response curve is consistently linear with a zero or analytically insignificant intercept, a calibration may be obtained using a single reference point standard. Ideally, there is a linear relationship extending from the origin through the calibration point. This single-point calibration offers savings in time and effort. The value for the intercept must be reported [5].

\subsubsection{Inverse Calibration}

The classical calibration above assumes no errors in $c$, but sample preparation nowadays may be less accurate than instrumental measurement. An inverse calibration is performed by using

$$
c=m y+b
$$

and then comparing with the classical calibration. Errors in sample preparation should be suspected if the results do not match up well [2]. Centner et al. demonstrated that inverse calibration yields more reliable predictions than classical calibration [6], and Grientschnig concluded that this was true regardless of the size of the calibration and test data sets [7]. 


\subsubsection{Standard AdDition}

The standard addition method is also known as the additive method, or simply as spiking. A known amount of the constituent being analyzed, the spike, is added to the sample to produce a larger instrumental response. For instance, a sample is analyzed to obtain an estimated result, a small concentrated amount of analyte equal to the amount presumed to be in the sample is added, and the sample is analyzed again to see if the response has doubled. A linear variation between concentration and response is assumed. The method is especially useful if an interfering substance is suspected, since its response will not change when the spike is introduced. The standard addition approach may also be used when a blank sample matrix (without analyte) can be obtained. Saxberg and Kowalski [8] developed and Kalivas [9] extended a generalized standard addition method using multiple linear regressions that allow for simultaneous analysis of different components in a mixture while accounting for interference.

\subsubsection{Internal Standards}

An internal standard is a substance that is similar (but not identical) to the analyte and is added to the sample. The ratio of the responses to the internal standard and the analyte is then compared to a calibration curve. The instrumental responses to the two must be distinguishable. Internal standards are often used when the scientist suspects a loss of analyte when the sample is prepared or when it is introduced into the instrument. A common internal standard in mass spectrometry is the deuterated version of the constituent of interest, since their responses are different, but possible losses before measurement should be identical. Internal standards are also useful when the analyte is not stable enough to be calibrated in other ways, although side reactions or other consequences could occur. Such effects took place when Álvarez del Pino et al. [10] compared internal and external standards for determining tannin in Spanish shrubs. They found that the slopes of the calibration lines were different when purified tannin was used as an internal or external standard, apparently because the internal standard reacted with other components in the sample.

\subsection{FUNDAMENTAL CRITERIA}

\subsubsection{Selectivity}

Whichever technique is selected for relating concentration with response, there are several factors that must be considered for the results to be valid. These include selectivity, range, accuracy, precision, detection limit, and quantitation limit. The selectivity of a method is its ability to measure the analyte in the sample matrix in the presence of other sample components. In chromatography, for instance, selectivity refers to the ability of a phase system to retain solutes to significantly different extents, resulting in analyte peaks that are completely resolved from other peaks. The term "specificity" is usually discouraged since it implies that nothing besides the analyte contributes to the result [11].

\subsubsection{ACCURACY}

The reliability of a method is based on its accuracy and precision. Accuracy, the difference between a measured value and the true value, is expressed in terms of error. A consistent error, such as one caused by an improperly prepared reagent, may produce replicate results that are similar but inaccurate by the same amount. This type of error is known as bias [2].

In a narrow sense, the only true values that may be known for certain are obtained in defined quantities and in counting discrete objects. All other measurements are obtained by comparison to a reference standard, such as one provided by NIST, or by comparison to another method known to be reliable. 


\subsubsection{Precision}

Precision is the amount of scatter in replicate measurements of the same quantity, and is expressed in terms of deviation. Measurement, sampling, and calibration errors all contribute to decreased precision and increased uncertainty [2]. Internal precision is measured by repeatability standard deviation, which reflects the results obtained on a test material by the same operator using the same method in the same laboratory with the same equipment within a short period of time. External precision is measured by reproducibility standard deviation and indicates the results obtained on a test material by different operators using the same method in different laboratories with different equipment [12]. In reporting results of a radical scavenging capacity assay for grains and flours, for example, Cheng et al. [13] expressed accuracy as percentage of recovery of the calibration standard and precision as intraday (same operator, same day) and interday (same operator, different days) variabilities.

\subsubsection{Detection Limit}

Instrumental noise consists of extraneous and unwanted signals which may result from thermal motions of electrons (Johnson-Nyquist noise), random fluctuations of current (shot noise), environmental factors, and other sources [14]. The detection limit, or minimum detectable value, is the lowest concentration of analyte that produces a signal that can be detected above instrumental noise. Usually, a signal-to-noise ratio $(\mathrm{S} / \mathrm{N})$ of at least 3 is required for a reportable result.

\subsubsection{Quantification Limit}

The quantification limit, also called the quantitation limit or minimum quantifiable value, is the lowest analyte level that can be measured with accuracy and precision. If not determined by experiment, it is often set as the concentration of analyte that leads to $\mathrm{S} / \mathrm{N}=10$ [15].

\subsubsection{RANGE}

The range of a method is the extent of concentrations within which accuracy and precision are retained and the relationship between concentration and response is constant. This frequently means that the calibration curve is linear between the lower and the upper concentration limits. Any results outside of the range would be invalid. The lower end of the calibration range is often the quantification limit.

A recent example of calibration and use of fundamental criteria is illustrated by a capillary electrophoretic study of olive oil by Carrasco-Pancorbo et al. [16]. Testing seven different analytes, they obtained linear calibration curves with the format of Equation 1.1, and determined recovery of other compounds by standard addition. They also calculated accuracy, internal precision (both intraday and interday), external precision, detection limit, quantification limit, and calibration range.

\subsection{OTHER CONSIDERATIONS}

\subsubsection{ROBUSTNESS}

When choosing a technique to be used for a particular analyte, the scientist should take into account the fundamental characteristics listed above [17]. Three other criteria, robustness, speed, and convenience, are also important. A technique demonstrates robustness or ruggedness if small changes in $\mathrm{pH}$, volume used in the analysis, or other parameters are within a specified tolerance $[3,15]$. As an example, Lai et al. [18] included instrumental drift, ambient temperature, and sample aging as robustness factors in their development of Fourier transform infrared spectroscopy for detection of vegetable oil adulteration. Their procedure was robust because minor changes in these variables did not significantly affect the accuracy and precision. 


\subsubsection{SPEED}

A necessary characteristic when choosing an analytical procedure is the amount of time required to complete it. Speed of analysis is less critical in a research laboratory, where relatively few samples are to be run, than in a continuous industrial process where timeliness is urgent. However, a time lag may be required between samples or groups of samples so that possible corrective actions may be taken.

\subsubsection{ConVenience}

Aspects of convenience include cost of purchasing and operating instruments, their availability when more than one analyst uses them, the sample size required, reagent stability and preparation time, staffing needs, and ease of performing the analytical method. Automatic sampling, if available, allows for unattended analyses and enables employees to attend to two or more tasks simultaneously.

\subsection{SUMMARY}

The results of an analytical study are only as good as the data used, but the data are only as good as the thoroughness displayed by the analyst in performing the measurements and minimizing errors. An analysis always takes less time to do once properly than to do over again because of carelessness or excessive speed.

\section{REFERENCES}

1. Elving, P.J. and Keinitz, H., Methodology of analytical chemistry, in Treatise on Analytical Chemistry, Vol. 1, 2nd ed., Kolthoff, I.M. and Elving, P.J. (Eds.), John Wiley \& Sons, New York, 1978, p. 53.

2. Brereton, R.G., Statistical assessment of results of food analysis, in Methods of Analysis of Food Components and Additives, Ötleş, S. (Ed.), Taylor \& Francis, Boca Raton, FL, 2005, Chap. 2.

3. Currie, L.A., Nomenclature in evaluation of analytical methods including detection and quantification capabilities, Pure Appl. Chem., 67, 1699, 1995.

4. Mitchell, D.G. et al., Multiple-curve procedure for improving precision with calibration-curve-based analyses, Anal. Chem., 49, 1655, 1977.

5. Cardone, M.J., Palermo, P.J., and Sybrandt, L.B., Potential error in single-point-ratio calibrations based on linear calibration curves with a significant intercept, Anal. Chem., 52, 1187, 1980.

6. Centner, V., Massart, D.L., and de Jong, S., Inverse calibration predicts better than classical calibration, Fresenius J. Anal. Chem., 361, 2, 1998.

7. Grientschnig, D., Relation between prediction errors of inverse and classical calibration, Fresenius J. Anal. Chem., 367, 497, 2000.

8. Saxberg, B.E.H. and Kowalski, B.R., Generalized standard addition method, Anal. Chem., 51, 1031, 1979.

9. Kalivas, J.H., Precision and stability for the generalized standard addition method, Anal. Chem., 55, 565, 1983.

10. Álvarez del Pino, M. et al., Comparison of biological and chemical methods, and internal and external standards, for assaying tannins in Spanish shrub species, J. Sci. Food Agric., 85, 583, 2005.

11. Vessman, J. et al., Selectivity in analytical chemistry, Pure Appl. Chem., 73, 1381, 2001.

12. ISO Standard 3534-1993, Statistics-Vocabulary and Symbols, International Organization for Standardization, Geneva, Switzerland, 1993.

13. Cheng, Z., Moore, J., and Yu, L., High-throughput DPPH radical scavenging capacity assay, J. Agric. Food Chem., 54, 7429, 2006.

14. Skoog, D.A. and West, D.M., in Principles of Instrumental Analysis, 2nd ed., Saunders College, Philadelphia, PA, 1980, p. 68.

15. Green, J.M., A practical guide to analytical method validation, Anal. Chem., 68, 305A, 1996. 
16. Carrasco-Pancorbo, A. et al., Rapid quantification of the phenolic fraction of Spanish virgin olive oils by capillary electrophoresis with UV detection, J. Agric. Food Chem., 54, 7984, 2006.

17. Tunick, M.H., Selection of techniques used in food analysis, in Methods of Analysis of Food Components and Additives, Ötleş, S. (Ed.), Taylor \& Francis, Boca Raton, FL, 2005, Chap. 1.

18. Lai, Y.W., Kemsley, E.K., and Wilson, R.H., Potential of Fourier transform infrared spectroscopy for the authentication of vegetable oils, J. Agric. Food Chem., 42, 1154, 1992. 


\title{
2 Microextraction Methods in Food Analysis
}

\author{
Kathy Ridgway, Sam P.D. Lalljie, and Roger M. Smith
}

\section{CONTENTS}

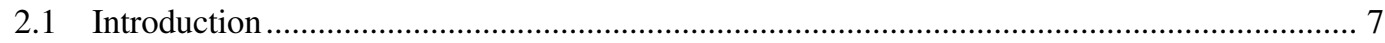

2.2 Theory

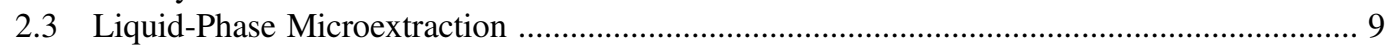

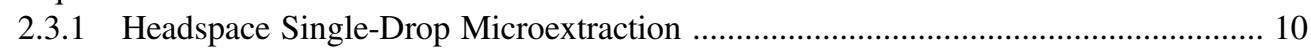

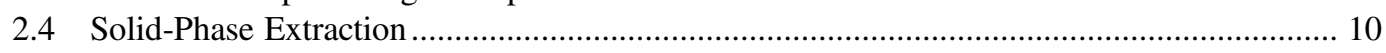

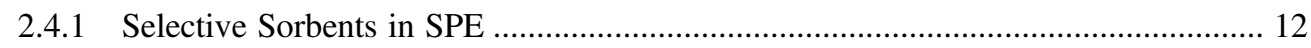

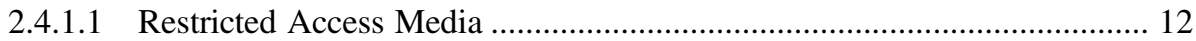

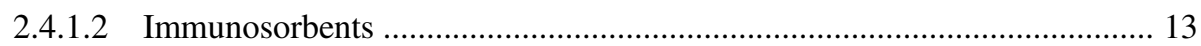

2.4.1.3 Molecularly Imprinted Polymers .......................................................... 14



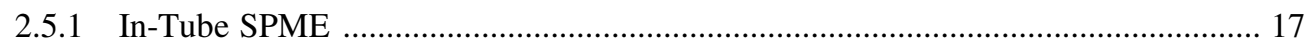

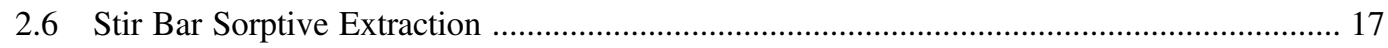

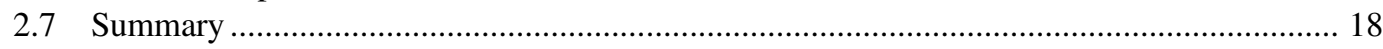

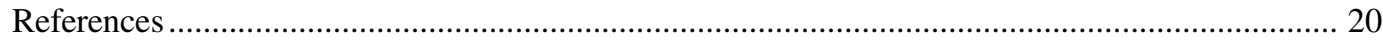

\subsection{INTRODUCTION}

The accurate determination of food components or residues and contaminants in food is necessary to ensure both the quality and safety of products to consumers. Methods of analysis must always be robust and accurate, but there is also the increasing demand on reducing the time spent on sample preparation and on using more environmentally friendly techniques that use smaller volumes of organic solvents.

Minimizing the number of steps in an analytical method results in a reduction not only in time but also in potential sources of error. Ease of automation of techniques is also becoming increasingly important to provide more robust and less labor intensive methods.

Food covers a wide range of materials, from solids such as cheese, viscous mixtures such as yogurt, and liquids, including wines and other drinks. The materials may be of natural plant or animal origin and be processed or manufactured. Within this wide range of complex matrices, the analytes may be present at high levels, typical for carbohydrates or fats, or be residues or contaminants at trace levels.

As a result, the methods of analysis often need to include extensive sample preparation before instrumental analysis to remove potential interferents, by separating the components of interest from unwanted matrix constituents, or to concentrate the analytes to enable detection at the low levels required.

To achieve these goals, a range of extraction and separation techniques have been employed to fractionate the sample, sometimes by a physical separation of vapors or liquids from solid materials but more frequently by employing a comprehensive or selective solvent extraction technique. 
Traditionally, concentration of the sample was achieved by evaporation of the extraction solvent until an analyte level suitable for instrumental analysis was obtained. This is wasteful both in the time of the analyst and in solvent usage.

This chapter will focus on those sample preparation techniques that provide microextraction/separation and concentration steps resulting in a final extract ready for instrumental analysis. In particular, it will examine techniques, such as liquid-phase microextraction (LPME), solid-phase extraction (SPE), and solid-phase microextraction (SPME), which can reduce the time spent on sample preparation and achieve the high concentration factors required for the determination of trace level components, residues, or contaminants in food. Some of the techniques follow an initial extraction stage and can be considered as cleanup/enrichment methods, whereas others offer combined extraction and enrichment in a single step.

Another technique that can be utilized to sample volatile analytes in food is headspace analysis. By sampling the headspace above a solid or liquid sample, usually after agitation and heating, a representative proportion of the volatile compounds are separated from the nonvolatile components, which remain in the sample matrix. Although direct static headspace will not be covered in this chapter, some of the techniques can be used to selectively extract analytes from the headspace above food samples, providing not only highly selective extraction, but also in some cases sample enrichment/concentration.

\subsection{THEORY}

In all extraction techniques, the transfer of analytes into the extracting phase (whether a gas, liquid, or solid) is dependent upon the chemical properties of the analytes. Key parameters that must be considered include volatility, solubility (hydrophobicity), molecular weight, ionizability $\left(\mathrm{p} K_{\mathrm{a}}\right)$, and polarity. The analyte is distributed between two immiscible phases and the distribution can be described in terms of equilibrium between these phases.

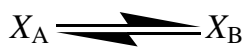

Therefore, using the Nernst distribution law, the distribution/partition coefficient $\left(K_{\mathrm{D}}\right)$ can be defined as

$$
K_{\mathrm{D}}=\frac{[X]_{\mathrm{A}}}{[X]_{\mathrm{B}}}
$$

where $[X]$ represents the concentration in each phase at constant temperature (or more accurately, the activity of the analyte in each phase). Usually, the total amount of all forms of the analyte present in each phase at equilibrium is considered.

If $K_{\mathrm{D}}$ is large, almost all the analyte is transferred into the extracting phase A in each extraction stage and extraction would be considered complete after two or three steps in traditional solvent (liquid-liquid) extraction.

A good guide to the distribution coefficient is the $n$-octanol/water partition coefficient $K_{\text {ow }}$ (also referred to as $P_{\text {ow }}$ or $P$ ) which is a measure of hydrophobicity (the compound's reluctance to enter a water phase).

$$
K_{\mathrm{ow}}=K_{\mathrm{D}}=\frac{[X]_{\mathrm{o}}}{[X]_{\mathrm{w}}}
$$

A compound with a larger value of $K_{\mathrm{ow}}$ is said to be more hydrophobic and will be easier to extract from water using an immiscible, usually organic, phase. Values of $K_{\mathrm{ow}}$ are often reported on a 
logarithmic scale $\left(\log K_{\mathrm{ow}}\right.$ or $\log P$ ) and generally a compound with a $\log P$ of 3 or above is considered as highly hydrophobic. In contrast, high water solubility is generally characterized by low hydrophobicity.

When considering the distribution of an ionizable analyte between phases, the acid dissociation constant $\left(\mathrm{p} K_{\mathrm{a}}\right)$ of a compound must also be considered and the $\mathrm{pH}$ at which an extraction is performed can be a key parameter.

Thus the efficiency of any extraction depends on the distribution ratio of the analyte between the phases and on the volume of each phase. If a large volume of extraction solvent is needed as in conventional liquid-liquid extraction, the extraction solvent may need to be evaporated in an extra step. To achieve a high concentration factor, ideally all the analyte of interest from a large volume of sample should be extracted into a small volume of extracting phase. The extracting phase can be in the form of a free liquid or a solid-supported liquid phase and the same principles of partition between the sample and the liquid-extraction phase apply. In the latter, the liquid-extraction phase can be coated on a fiber or a solid surface. Alternatively, the extraction phase can be a solid sorbent where extraction is based on the interaction at the surface only (adsorption). This method is employed in some SPE methods, where the sample or solution is passed through a column and analytes can be exhaustively extracted onto the sorbent.

Some methods are not intended to provide exhaustive extraction and are optimized at the equilibrium point of the phase distribution. These include SPME and stir bar sorptive extraction (SBSE) and can involve mechanisms based on both partitioning, where the analytes are partitioned into the matrix and are retained in the bulk phase as in liquid-liquid extraction, and adsorption, where the analyte concentrates onto the surface only. Different mechanisms can be employed depending on the analytes of interest and conditions must be optimized for each application and different food matrix.

\subsection{LIQUID-PHASE MICROEXTRACTION}

Miniaturized versions of liquid-liquid extraction have been devised, including LPME, in which the analyte partitions between the bulk aqueous phase and a very small volume of organic solvent. The extraction can be performed in different modes, including static, dynamic, and headspace LPMEs. This technique was first introduced in 1996 [1] and was subsequently reviewed by Wood et al. [2] and by Psillakis and Kalogerakis [3], including a useful comparison with SBSE and SPME (discussed later).

Recent developments use only a single droplet of the extraction solvent (single-drop microextraction, SDME), which is suspended at the tip of a needle and exposed to the sample solution. As the extracting phase in this approach is typically only microliters of solvent, large concentration factors are possible even with relatively small sample sizes (a few milliliters). Although the method has potential for liquid samples, reported food applications are limited, but Zhao et al. [4] recently reported an SDME for the analysis of organophosphorus pesticides in orange juice.

However, the droplet can only be used with care because it is not rigidly held in position. To overcome this problem, a porous hollow fiber membrane can be used to support the organic solvent during the extraction from the aqueous sample. This approach has been reviewed by Rasmussen and Pedersen-Bjergaard [5]. The fiber allows the use of vigorous stirring or agitation without loss of the microextract (as can occur in droplet LPME) and as a fresh hollow fiber can be used for each extraction, any carryover is avoided. The hollow fiber, because of the pores in its walls, also shows some selectivity, preventing the extraction of higher molecular weight materials. This technique has been referred to as hollow fiber protected liquid-phase microextraction (HF-LPME). Food applications are limited although LPME using a hollow fiber membrane was used for the determination of ochratoxin $\mathrm{A}$ in wine [6]. The technique has also been applied to human breast milk [7] and bovine milk [8] (Figure 2.1), but centrifugation of the samples before extraction was necessary to improve analyte extractability. Low recoveries were obtained due to strong analyte interactions with the matrix. 


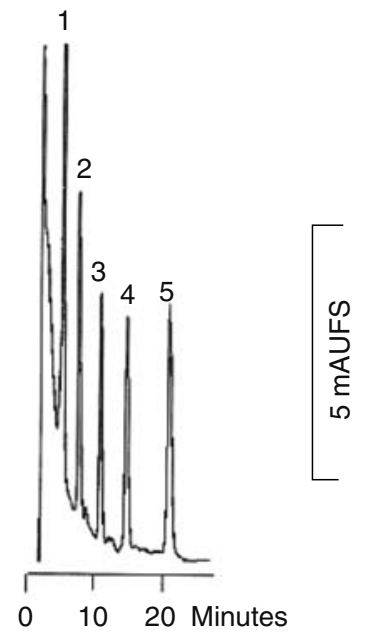

FIGURE 2.1 Pesticides of phenoxy acid herbicides extracted from milk sample spiked at $10 \mathrm{ng} / \mathrm{mL}$. Extraction conditions: 1-octanol as the impregnation solvent, $0.5 \mathrm{M} \mathrm{HCl}$ in donor phase, $0.1 \mathrm{M} \mathrm{NaOH}$ in acceptor phase, extracted for $60 \mathrm{~min}$ at $1250 \mathrm{rev} / \mathrm{min}$ peak identification: (1) 2,4-DCBA (2,4-dichlorobenzoic acid), (2) 2,4-D (2,4-dichlorophenoxyacteic acid), (3) mecoprop (2-(4-chlorophenoxy)-2-methylpropionic acid), (4) 3,5-DCBA (3,5-dichlorobenzoic acid), (5) fenoprop (2-(2,4,5-trichlorophenoxy) propionic acid), mAUFS (milli Absorbance Units full scale). (Reproduced from Zhu, L., Huey Ee, K., Zhao, L., and Lee, H.K., J. Chromatogr. A, 963, 335, 2002. With permission.)

Automation of SDME for liquid samples is difficult, and any agitation of the sample must be carefully controlled to avoid loss of the extracting solvent. However, some manufacturers now provide membrane inserts for in-vial extraction that could make full automation of HF-LPME possible for some samples.

\subsubsection{Headspace Single-Drop Microextraction}

In a similar way to the use of droplet liquid-liquid extraction, a single drop of solvent suspended from the tip of a syringe can be used to extract the headspace of a sample [2]. This technique has been used for residual solvent analysis, such as aromatic hydrocarbon and chlorinated solvents in edible oils and pharmaceutical products [9]. The extraction solvent must have a boiling point which is high enough to avoid evaporation during sampling. The use of an internal standard is recommended if the method is performed manually. An automated method has been reported as reasonably robust, although there was some evidence that the extracting drop had fallen off the needle on a few occasions. Practical difficulties include a limited choice of solvents because of the viscosity that is required, and further work is needed to prove the reproducibility of this technique.

\subsection{SOLID-PHASE EXTRACTION}

SPE involves the partition of analytes between a solid sorbent (extracting phase) usually held in a short column and the sample matrix (liquid phase). To ensure efficient extraction, the affinity of the analytes for the solid phase must be greater than that for the sample matrix. A comprehensive review covering trends, method development, coupling with liquid chromatography, and all types of SPE sorbent was published by Hennion in 1999 [10] and a number of books have looked at the theory and application of the technique in detail [11,12]. Theoretical aspects of SPE are covered in detail by Poole et al. [13], who discussed the use of computer-aided method development and method optimization.

The technique usually involves three or four steps, as illustrated in Figure 2.2. 


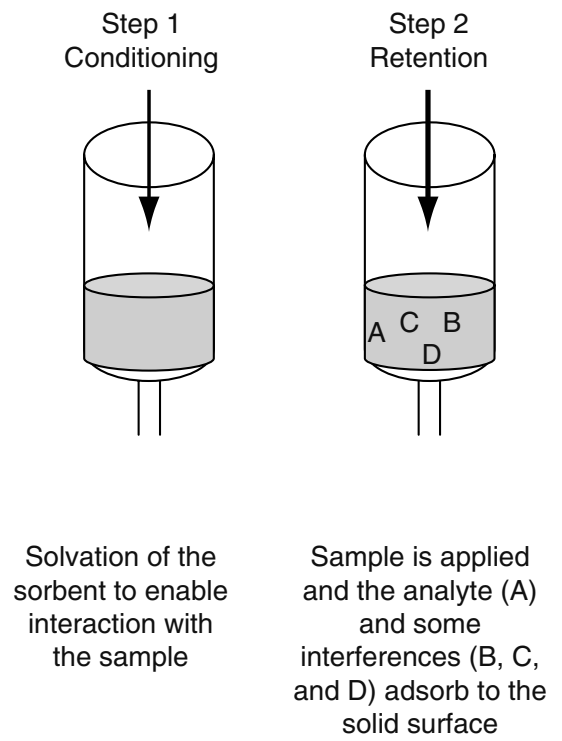

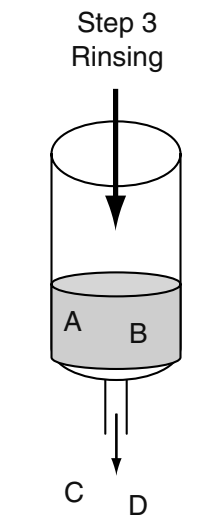
Selective washing to remove interferences/ unwanted compounds

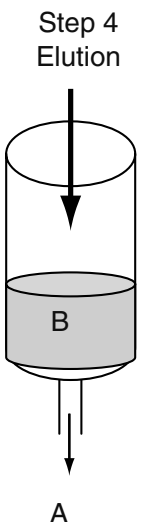
Selective desorption and collection of analytes for analysis

FIGURE 2.2 Solid-phase extraction.

The sample is loaded onto a pretreated column or cartridge filled with the required sorbent which traps the analytes and allows most of the matrix, usually an aqueous solution, to pass to waste. After a rinse step, the analyte of interest is eluted with a small volume of a suitable solvent giving a concentrated extract and leaving insoluble interferences on the column. SPE is considered to be an exhaustive technique as the retention of analytes on a sorbent is based on chromatographic retentions where all the analyte is removed from the sample (and subsequently eluted). The sorption process must be reversible. The selectivity of trapping and elution can be obtained by adjusting the $\mathrm{pH}$ and solvent polarity.

The choice of sorbent in the cartridge is dependent on the food matrix and analyte(s) of interest. Numerous sorbent materials are available using different mechanisms for extraction/retention of analytes, including partitioning, adsorption, and ion exchange interactions based on van der Waals, polar/dipole-dipole, hydrogen-bonding, or electrostatic (ion exchange) interactions. Typical materials include silica bonded with nonpolar alkyl chains, especially C18 (octadecyl) and C8 (octyl) groups or polar chains such as hydroxyl and cyano groups. Other support materials are polymeric resins (polystyrene/divinyl benzene copolymer), Florisil (activated magnesium silicate), and polar sorbents, such as alumina, charcoal, and unbonded silica. Ionic functional groups, such as carboxylic acid or amino groups can also be bonded to silica or polymeric supports to create ion exchange sorbents. Some cartridges use mixed-mode sorbents that use both primary and secondary mechanisms for selective retention of analytes and some very specific selective sorbents have been designed (Section 2.4.1).

The most common SPE system is the syringe barrel cartridge, but thin-porous glass fibers, thin-coated glass fibers, PTFE (polytetrafluoroethylene) disks embedded with sorbents, and disposable plastic pipette tips fitted with sorbent beds are all available. One of the drawbacks of SPE is that the packing must be uniform to avoid poor efficiency and automated systems can have difficulties with reproducibility for some sample types. Analyte sorption is dependent on both the sample volume and sorbent mass, and the theoretical aspects have been reviewed [14].

The presence of particulate matter in the sample can affect the sorption process and, in some cases, filtration of the sample before SPE may be necessary. The sample matrix can also affect the ability of the sorbent to extract the analyte owing to competition for retention. Many traditional sorbents, such as C18 silica, are limited in terms of selectivity and insufficient retention of very 


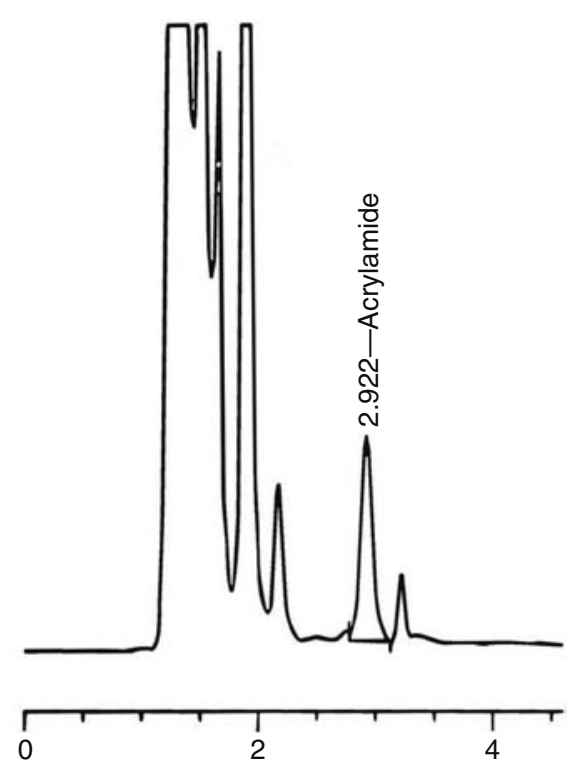

FIGURE 2.3 Liquid chromatography/ultraviolet (LC/UV) chromatogram of acrylamide in a French fries extract using Strata-X-C. LC Conditions: Synergi Polar-R $4 \mu 150 \times 3.0 \mathrm{~mm}$, mobile phase 94:6 (V:V) water: acetonitrile at $0.4 \mathrm{~mL} / \mathrm{min}$, injection volume: $10 \mu \mathrm{L}$. (Reproduced from Peng, L., Farkas, T., Loo, L., Dixon, A., Teuscher, J., and Kallury, K., Rapid and Reproducible Extraction of Acrylamide in French Fries Using a Single SPE Sorbent-Strata-X-C, Phenomenex, Inc., Torrance, CA, 2007. With permission.)

polar compounds can be a problem. The use of hydrophilic materials for the improved extraction of the most polar compounds by SPE was detailed by Fontanals et al. [15]. More recently, as well as the development of more selective sorbents, the use of monolithic columns and multiwalled carbon nanotubes (MWCNTs) has been investigated [16] for the determination of polybrominated diphenyl ethers in water and milk.

Typical examples of the use of SPE in food analysis were given in a review in 2002 [17], and include the determination of folic acid in fruit juices [18], and antioxidants in margarine [19]. More recent examples include the determination of amines in beer [20], veterinary drugs in shrimp [21], acrylamide in French fries [22] (Figure 2.3), and heterocyclic amines in meat [23].

In recent years, automation of SPE has become more widely available and several online systems are now available. Although, in most cases, and particularly for solid or semisolid foods, an initial extraction step is required before cleanup/extraction/concentration by SPE. Carbon-based solid phase extraction tubes have been used for extraction from fruit and vegetables (Figure 2.4).

\subsubsection{Selective Sorbents in SPE}

Most SPE methods are based on the trapping of compounds falling into a broad polarity region, but lack specificity for selected compounds. More specific extraction media have been developed to either use two mechanisms in conjunction, as in restricted access media (RAM), or to employ biological specificity (affinity columns) or their synthetic mimics (molecularly imprinted polymers, MIPs) to trap specific groups of compounds of interest.

\subsubsection{Restricted Access Media}

The RAM sorbents [24] for SPE were developed particularly for the analysis of biological samples, such as plasma and serum, as they are designed to exclude macromolecules, such as proteins, and 


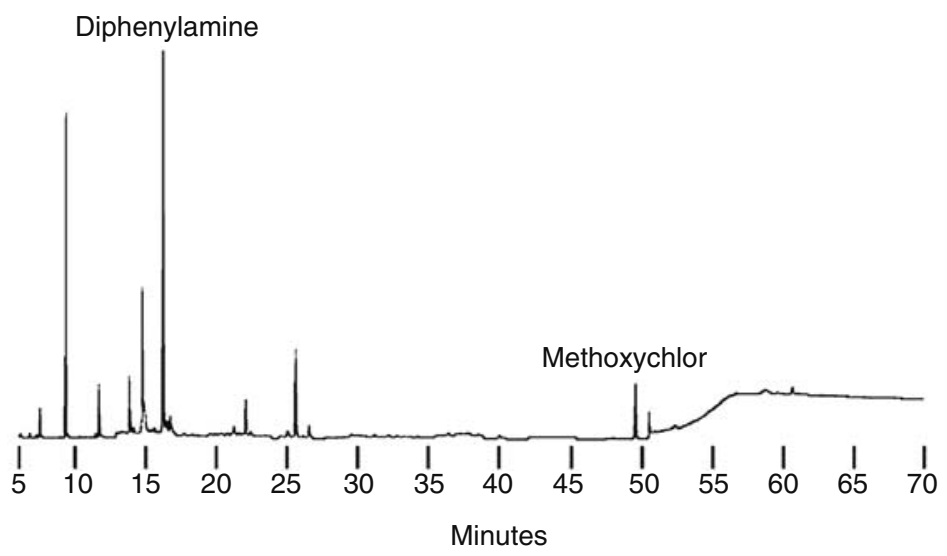

FIGURE 2.4 Extraction of pesticides from homogenized fruit. Sample $(50 \mathrm{~g})$ homogenized with acetonitrile $(100 \mathrm{~mL})$ and $10 \mathrm{~g}$ for $5 \mathrm{~min}$. Following concentration, extracted with ENVI-Carb, $6 \mathrm{~mL}, 500 \mathrm{mg}$ SPE tube. Pesticides eluted with acetontirile:toluene (3:1) and extract concentrated with acetone. GC column $14 \%$ cyanipropylphenyl $/ 86 \%$ dimethylsiloxane, $30 \mathrm{~m} \times 0.25 \mathrm{~mm}$ ID $0.15 \mu \mathrm{m}$ film. Oven $70^{\circ} \mathrm{C}(2 \mathrm{~min})$ to $130^{\circ} \mathrm{C}$ at $25^{\circ} \mathrm{C} / \mathrm{min}$ to $220^{\circ} \mathrm{C}$ at $2^{\circ} \mathrm{C} / \mathrm{min}$ to $280^{\circ} \mathrm{C}$ at $10^{\circ} \mathrm{C} / \mathrm{min}$, held for $4.6 \mathrm{~min}$. Carrier helium, Mass selective detector (MSD) $\left(285^{\circ} \mathrm{C}\right)$, injector in splitless $(2 \mu \mathrm{L})$. (Reproduced from Supelco Web site-Bulletin 900 . With permission.)

allow the trapping of smaller drug molecules. They combine the exclusion of proteins and other high-molecular mass matrix components with the simultaneous enrichment of low-molecular mass analytes at the inner pore surface. Macromolecules are excluded either by a physical barrier (pore diameter) or by a chemical diffusion barrier created by a protein network at the outer surface of the particle. Various RAM sorbents are available with different surface chemistries [25]. Internal surface reversed phase (ISRP) supports are the most popular in which a C4-, C8-, or C18-bonded reversed phase covers the internal pore surface of a glyceryl-modified silica. The interaction sites within the pores are only accessible to small molecules and the analytes are retained by conventional SPE retention mechanisms, such as hydrophobic or electrostatic interactions.

Several food applications are given in a review by Souverain et al. [25], including the direct analysis of pharmaceuticals in milk [26] and tissue [27].

\subsubsection{Immunosorbents}

Molecular recognition can be used to create highly selective immunosorbents by linking an antibody to a solid support (such as silica), which is then packed into an SPE cartridge or precolumn. This technique uses the very specific interactions between analytes and a biological system to enable the selective retention of the compounds of interest. The analyte can then be released by elution with solvent or a change in $\mathrm{pH}$. The technique is particularly suited to complex biological and environmental samples. The selectivity is based on the antigen-antibody interaction and immunosorbents can be designed for single analytes. Some antibodies can also bind to other analytes with similar structures to the antigen (known as cross-reactivity), and this can be utilized to develop classselective sorbents. One of the major disadvantages of this technique is the need to initially develop the antibody, which makes it impractical for one-off analyses. The analyte-antibody interaction can also be affected by the sample matrix, leading to low extraction recoveries. A review by Hennion and Pichon [28] describes immuno-based extraction sorbents and also the use of artificial antibodies.

Examples of the use of immunosorbents for food analysis include the determination of pesticides (imazalil and phenylurea herbicides) in fruit juices [29,30]. Methods for the analysis of mycotoxins are now commercially available and methods have been developed and accepted as 
valid for several food matrices, including peanut butter, roast coffee, and baby food [31]. Immunosorbents have also been developed for some veterinary drugs, such as fluoroquinolones in chicken liver [32] or corticosteroids in animal feed [33].

\subsubsection{Molecularly Imprinted Polymers}

Attempts have been made to mimic the specificity of immunological products with synthetic MIPs. MIPs are created by forming a polymer structure containing selected functional groups, around a template analyte molecule. After removal of the template, the polymer contains highly stable cavities with active sites which are specific to the shape and functionality of the analyte of interest. Trapping and retention of analytes is due to the shape recognition in the cavities and interactions, such as hydrogen bonding, and hydrophobic interactions.

MIP-SPE sorbents allow for larger sample volumes to be used than conventional SPE materials because of their selectivity. They can be heated and are stable in both organic solvents and strong acids and bases, unlike many immunosorbents. However, a separate MIP must be made for each analyte, although they can sometimes perform group trapping if all the analytes contain a common structural feature that has formed the active feature of the template. Because of the nature of their selectivity, once developed, MIPs can often be used for a number of matrices, even though the interaction may be different. MIP-SPE can be used both online and off-line. Coupled with HPLC (high-performance liquid chromatography), the MIP can be packed in a cartridge and used via column switching, before analysis [34,35].

One problem encountered with MIPs is that the selective interactions, which were present in the organic solvent in which the template was prepared, do not always work as well in aqueous solutions. However, it is possible to overcome this problem, by initially retaining the sample by a nonselective interaction, then washing the cartridge with an organic solvent to trap the analyte using selective binding. Alternatively, the analyte can be transferred from an aqueous sample or extract into an organic solvent before SPE.

MIPs have been used as selective sorbents for a range of analytes and matrices [36-38]. Food applications to date are limited, but include the determination of triazines in liver [39], nicotine in chewing gum [40], and the detection of Sudan I as a contaminant in food matrices [41]. Currently, the time taken to develop and produce such sorbents is the rate limiting step for new and emerging food contaminants.

\subsection{SOLID-PHASE MICROEXTRACTION}

Although SPE and related methods minimize the use of solvents, they still require an elution stage which effectively dilutes the extract. In contrast, SPME is a solvent-free sample preparation technique. It uses a fused silica fiber coated with an appropriate stationary phase as the extraction medium attached to a modified micro-syringe. The sample is usually released by thermal desorption directly into the injection port of a gas chromatography (GC), but can also be released into an HPLC mobile phase. It was originally developed by Arthur and Pawliszyn [42] in 1990 and a number of books are available on this technique [43-45]. The main advantages of SPME are the combination of sampling and extraction into one step and the ability to examine small sample sizes. It can also have high sensitivity and can be used for polar and nonpolar analytes in a wide range of matrices.

SPME is essentially a two-step process. Firstly, the partitioning of analytes between the sample matrix, which can be a liquid sample or headspace, and the fiber coating, and then the desorption of the (concentrated) extract from the fiber into the analytical instrument. The physical and chemical properties of the extracting phase on the fiber and the target analyte molecular weight, volatility, and polarity determine the partition coefficient of the analyte between the fiber coating and the sample matrix. For high extraction efficiency, the polarity of the phase should match that of the analyte and 
the amount of analyte extracted onto the fiber depends on the polarity and thickness of the polymer phase, the extraction time, and the concentration of the analyte in the sample.

The yield also depends on the properties of the sample matrix, but generally SPME of the analyte from the matrix is not exhaustive. The maximum sensitivity would be obtained when equilibrium is reached; however, extractions can instead be performed for a defined period of time as long as the yield at that time is reproducible [46]. The speed of extraction can be improved by agitation of the matrix and the equilibrium can be altered by the addition of salt or by changing the $\mathrm{pH}$ or temperature.

A fiber with a thicker coating is best to retain volatile analytes and transfer them to the GC injection port without loss, but a thin coating is used to ensure a rapid release of higher boiling point compounds during thermal desorption. Fibers with different thickness and polarities are available and can generally be classified into two groups: pure liquid polymer coatings, such as PDMS (polydimethylsiloxane) and PA (polyacrylate), and mixed films containing liquid polymers and solid particles, such as Carboxen-PDMS and divinylbenzene (DVB)-PDMS. Extraction can be based on absorption (as with liquid phases, such as PDMS), or adsorption on the surface of the polymer (as with more rigid polymeric structures, such as polystyrene-DVB phases). Mixed films combine the absorption properties of the liquid polymer with the adsorption properties of the porous particles, but these phases generally have a more limited lifetime.

PDMS is strongly hydrophobic and is particularly suitable for extraction from aqueous matrices. It is a commonly used phase with a generic selectivity for many types of nonpolar analytes. PA and Carbowax (CW)-DVB are better for more polar analytes, such as phenols or alcohols. Carboxen acts as a carbon molecular sieve and is often used in combination with PDMS (Carboxen-PDMS) for lowmolecular weight polar analytes. It is generally better than PDMS, but can give a poorer reproducibility and take longer to equilibrate. DVB is a solid polymer, with slightly larger pores than Carboxen and in combination with PDMS is best suited to semi-polar analytes. More recent papers also detail the production of SPME materials with new solgel coatings. These phases are reported to exhibit high thermal stability and tolerance to organic solvents. Other new coating materials include affinity coatings for target analytes and chiral coatings for optically active analytes [46].

Fibers can be reused and manufacturers claim that under most conditions fibers can provide 50-100 extractions. However, in practice, the fibers can be fragile and can either be broken or the coating can be damaged during injection or agitation. Extractable but nonvolatile compounds in the sample can remain on the fiber, which can limit the fiber's lifetime and reproducibility. Proteins can also adsorb irreversibly to the fiber, changing the fiber properties and making it unusable for more than one sample. Problems with batch to batch variation of fiber coatings have also been reported. A recent development is that of superelastic SPME where the fiber is a metal alloy with elastic properties and can be coated with PDMS/DVB, Carboxen/PDMS, and DVB/Carboxen-PDMS as well as PDMS [47]. This improves the robustness and overcomes problems caused by the fibers breaking due to misalignment with injection ports or in viscous matrices. To achieve the required throughput with multiple samples automated SPME systems can be used.

The extraction temperature, time, and sample agitation rate must be optimized for each application and operating conditions must be consistent. Because of matrix effects, quantitation generally requires matrix matched standards or the method of standard additions can be used. The use of an isotopically labelled internal standard should be considered. The analyte concentration can also influence the extraction. At low concentrations $(<50 \mathrm{ppb})$, the equilibrium is concentration dependent, so changes in sample volume do not affect response. However, at higher concentrations, volumes become significant and must be consistent for samples and standards, especially for compounds with high distribution coefficients. The presence of high concentrations of other matrix components in the sample can result in competitive binding and displacement. In some cases, such as alcoholic beverages, because the ethanol is a competing solvent, the levels can alter the distribution constant. 
Extraction can be performed by direct immersion of the fiber into liquid samples, by extraction of the headspace above the sample, or by using a membrane protected fiber. Direct immersion SPME into complex matrices, such as many foods can be difficult, as the fiber can be damaged and is therefore more suited to semi- or less-volatile analytes in liquid samples or solutions. For dirty samples, the SPME fiber can be rinsed to remove interferences after extraction.

Headspace sampling is particularly suitable for many complex food matrices, as the nonvolatile components do not come into contact with the fiber and the method can be used with both solid and liquid matrices. This mode of extraction is based on the equilibrium between three phases: the matrix, the headspace, and the fiber. Effectively the distribution is between the fiber and the matrix. Although raising the temperature increases the volatility of the analyte, it may result in less deposition onto the fiber as in the headspace-fiber equilibrium, the analyte will again favor the vapor phase. Thus headspace SPME offers a different selectivity as it favors the less volatile compounds in contrast to direct headspace, which favors the more volatile components [48]. Equilibrium is reached faster in headspace analysis than direct fiber insertion into the liquid matrix because of better mass transfer. Typical analytes include aromas (Figure 2.5), flavors, and fragrance components [48-53].

An alternative for dirty liquid samples is to protect the fiber by placing it inside a hollow cellulose membrane. This can have an added size exclusion effect (e.g., only allowing compounds with molecular weight less than $1000 \mathrm{Da}$ to diffuse through the membrane). However, using this technique requires a much longer extraction time [54], and clogging of the membrane would be an issue for many food matrices.

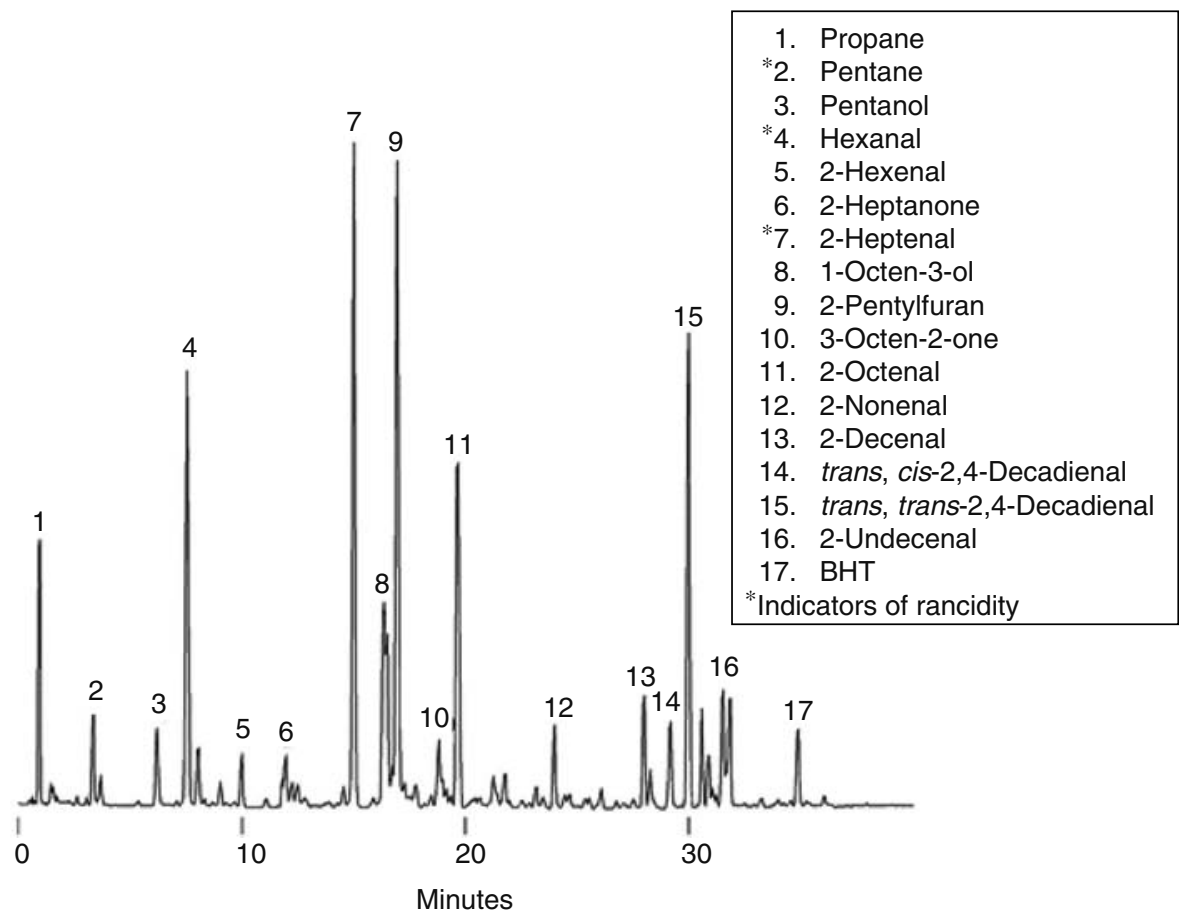

FIGURE 2.5 Flavor compounds extracted from rancid corn oil. SPME fiber PDMS, $100 \mu \mathrm{m}$ film, headspace sampling ( $45 \mathrm{~min}, 40^{\circ} \mathrm{C}$ ), desorption $250^{\circ} \mathrm{C}, 1.5 \mathrm{~min}$. GC column SPB-5, $30 \mathrm{~m} \times 0.53 \mathrm{~mm}$ ID $5 \mu \mathrm{m}$ film. Oven $40^{\circ} \mathrm{C}(5 \mathrm{~min})$ to $220^{\circ} \mathrm{C}$ at $4^{\circ} \mathrm{C} / \mathrm{min}$. Carrier helium, $5 \mathrm{~mL} / \mathrm{min}$ Flame Ionisation detector (FID) $\left(300^{\circ} \mathrm{C}\right)$, injector in splitless $(1 \mathrm{~min}), 250^{\circ} \mathrm{C}$. (Reproduced from Supelco Web site-SPME application note. With permission.) 
In general, SPME provides low recoveries because of the small volume of the stationary phase that can be bound to the fiber. It needs to be calibrated carefully to achieve accurate and reproducible quantitative measurements.

Derivatization can be used to overcome low extraction efficiencies for certain volatile, polar, or thermally unstable analytes, but is mainly used to improve chromatographic behavior. It can be performed before, during, or after the extraction procedure. Typical reactions include the conversion of fatty acids to their methyl esters or addition of functional groups, such as pentafluorobenzene, to enhance detection and the field has been reviewed by Stashenko and Martinez [55].

Most SPME methods use GC as the instrument, but it is also possible to interface the method with HPLC using solvent desorption, which can take place in a static or dynamic (flowing eluent) mode. The static mode is preferred for more strongly adsorbed analytes, as the fiber is soaked in the mobile phase, or another solvent, for a specified time, before injection. For automation, in-tube SPME devices (Section 2.5.1) are more suited to HPLC applications. Fiber SPME-HPLC can lead to peak broadening if analytes are slow to desorb, but with in-tube devices the analytes are desorbed before injection.

Reviews of the application of SPME in food analysis by Kataoka et al. [46] and by Wardencki et al. [56] give many examples of applications and techniques. These include food components, such as volatile aroma compounds and fatty acids [46], and flavor analysis [51] where commonly headspace-SPME is the preferred method. Recent examples include the determination of furan in baby food [57] and formaldehyde in fish [58].

\subsubsection{IN-TUBE SPME}

An alternative to the externally coated SPME fiber is an internally coated capillary, through which the sample flows, or is drawn repeatedly, and analytes are then eluted or desorbed. This technique was developed due to the difficulties of trying to interface SPME with HPLC systems [59]. Several in-tube SPME options can be used with LC, which are suitable for automation and can continuously perform extraction, desorption, and injection. The capillary extraction tube is placed between the injection loop and the injection needle of the HPLC autosampler. A disadvantage of in-tube devices is that particles need to be removed from samples before extraction (by filtration or centrifugation). The amount of analyte extracted by the phase depends on the polarity of the capillary coating, the number and volume of extraction (draw/eject) cycles, and the sample $\mathrm{pH}$. As would be expected, target analytes with lower $K$ values need longer equilibration times. If too many extraction cycles are performed peak broadening can occur.

A variant of in-tube SPME termed solid-phase dynamic extraction (SPDE) using wall-coated needles was described by Lipinski [60] for the extraction of liquid samples.

Using an adapted syringe needle allows for dynamic extraction, providing high concentration factors and a variety of sorbents are now available.

Kataoka [61] reviewed automated in-tube SPME, giving applications for both food contaminants and food component analysis. Examples included heterocyclic amines in beefsteak [62] and endocrine disruptors in fatty foods [63].

\subsection{STIR BAR SORPTIVE EXTRACTION}

SBSE was developed by Baltussen et al. [64] to overcome the small extraction medium volumes used in SPME. Instead of a coated fiber, a glass stirrer bar is coated with a bonded adsorbent layer (PDMS) to give a larger volume of the stationary phase, which is generally more robust than SPME fibers.

Extraction is achieved by sorption onto the PDMS coating and transfer from the stirrer bar to a GC is then achieved either by thermal desorption or, for HPLC, by elution with a solvent. Because of the larger volumes of PDMS on the stir bar, higher concentration factors with longer extraction times can be achieved. The extraction mechanism from aqueous solutions is based on an absorption 


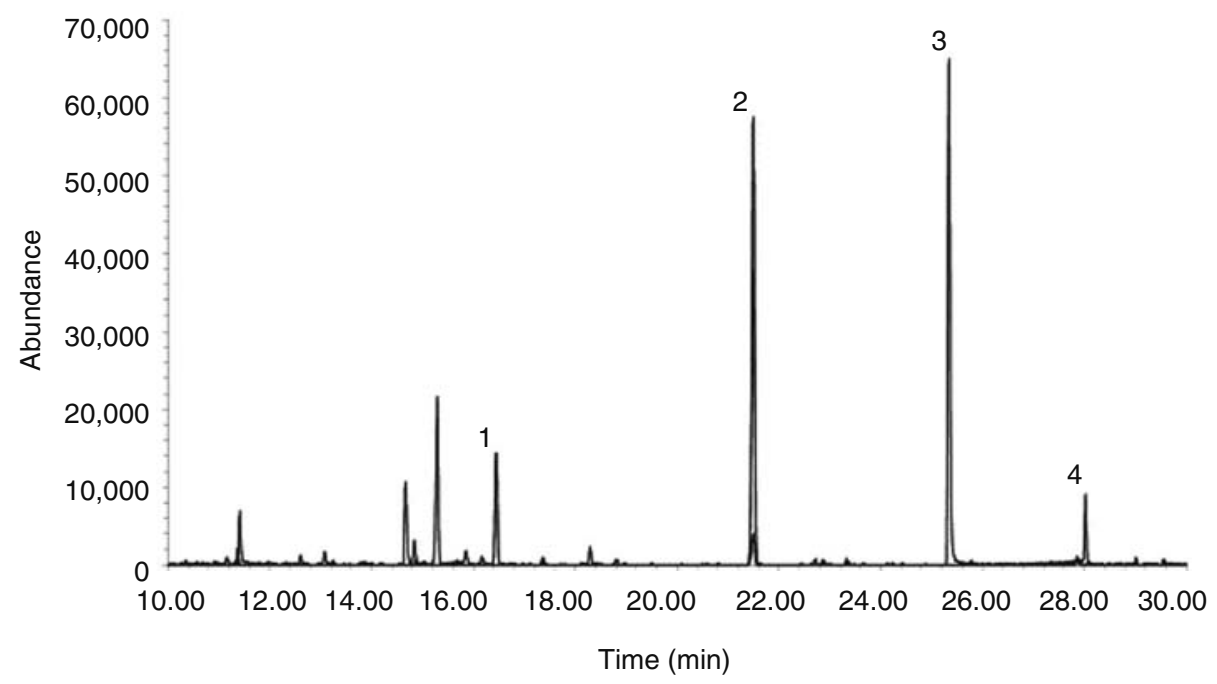

FIGURE 2.6 Extracted ion chromatogram at $m / z$ 187, 283, and 285 of the (SBSE-Thermal desorption GC-MS) analysis of Italian sparkling wine: (1) vinclozolin, (2) procymidone, (3) (3,5-dichlorophenyl)hydantoin, and (4) iprodione. (Reproduced from Bicchi, C., Cordew, C., Iori, C., Rubiolo, P., and Sandra, P., HRC J. High Resolut. Chromatogr., 23, 539, 2000. With permission.)

process and the octanol/water distribution coefficients can be used to predict recoveries. The technique is generally suited to compounds with a $\log P>2\left(K_{\mathrm{ow}}>100\right)$.

It can be used directly in liquid or semisolid complex matrices, such as yogurt, and as with SPME, the stir bar can also be used to sample the volatiles and semi-volatiles in the headspace above the sample. Derivatization can again be used to extend the applicability of the technique, and multiple stir bars can be used to improve sensitivity. The technique is commercialized under the name twister. Currently, only a PDMS coating is commercially available, making the technique most suited to nonpolar analytes from aqueous media.

Dual-phase stir bars have been described by Bicchi et al. [65], which consist of a short PDMS tube with an inner cavity that is packed with activated carbon adsorbent. This method combines both sorption and adsorption simultaneously and improved the recovery of volatile or polar compounds when compared to conventional PDMS stir bars for the analysis of coffee and sage (by headspace) and whisky (by immersion).

The use of SBSE for food analysis is increasing and the technique has been used for the analysis of coffee brew [50], alcoholic beverages [51], the determination of pesticides in fruits [66] and wine [67] (Figure 2.6), and for examining the headspace of aromatic and medicinal plants [68] and food [69].

\subsection{SUMMARY}

Microextraction methods provide highly selective techniques that are either solvent free or use only small volumes of solvent. They can be used to provide robust, accurate methods that are necessary for food analysis to ensure both the quality and safety of products and ingredients. They require much smaller sample sizes than conventional extraction methods and often enable a selective extraction or extract cleanup technique that can provide enrichment of analytes from complex matrices and thus enable detection down to the levels required for food safety and quality. Many of the approaches can be automated to enable the use of high-throughput methods that are simple, reliable, and more environmentally friendly. A summary of food applications of the techniques described in this chapter is given in Table 2.1. 
TABLE 2.1

\section{Food Applications of Microextraction Techniques}

\begin{tabular}{|c|c|c|c|}
\hline \multirow[b]{2}{*}{ Food Type } & \multicolumn{2}{|c|}{ Liquid-Phase Microextraction } & \multirow[b]{2}{*}{ References } \\
\hline & Analytes & Technique & \\
\hline Orange juice & Organophosphorus pesticides & SDME & [4] \\
\hline Wine & Ochratoxin A & HF-LPME & [6] \\
\hline Human breast milk & Basic drugs & HF-LPME & [7] \\
\hline Bovine milk & Phenoxy herbicides & HF-LPME & [8] \\
\hline Beer & Alcohols & Headspace-LPME & [71] \\
\hline \multicolumn{4}{|c|}{ Solid-Phase Extraction } \\
\hline Beer & Amines & SPE & [20] \\
\hline Shrimp & Veterinary drugs (multi-class) & SPE & [21] \\
\hline Animal tissues & Fluoroquinolone residues & SPE & [72] \\
\hline Meat & Heterocyclic amines & SPE & [23] \\
\hline Milk & Pharmaceuticals & SPE (RAM) & [26] \\
\hline Tissue & $\begin{array}{l}\text { Pharmaceuticals (including nicardipine, } \\
\text { nitrendipine, felodipine, and benzodiazepines) }\end{array}$ & SPE (RAM) & [27] \\
\hline Fruit juice & Drugs & SPE-Immunosorbents & [29] \\
\hline $\begin{array}{l}\text { Peanut butter, pistachios, } \\
\text { fig paste, and paprika }\end{array}$ & Aflatoxins (B1,B2,G1,G2) & SPE-Immunosorbents & {$[31]$} \\
\hline Baby food & Aflatoxin B1 & SPE-Immunosorbents & [31] \\
\hline Milk & Aflatoxin $\mathrm{m} 1$ & SPE-Immunosorbents & [31] \\
\hline Roast coffee and baby food & Ochratoxin A & SPE-Immunosorbents & [31] \\
\hline Apple juice and puree & Patulin & SPE & [31] \\
\hline Chicken liver & $\begin{array}{l}\text { Fluoroquinolones (ciprofloxacin, enrofloxacin, } \\
\text { sarafloxacin, and difloxacin) }\end{array}$ & SPE-Immunosorbents & [32] \\
\hline Animal feed (and urine) & $\begin{array}{l}\text { Corticosteroids (dexamethasone, } \\
\text { flumethasone, and triamcinolone) }\end{array}$ & SPE-Immunosorbents & [33] \\
\hline Liver & Triazines & SPE-MIPs & [39] \\
\hline Chewing gum & Nicotine & SPE-MIPs & [40] \\
\hline \multicolumn{4}{|c|}{ Solid-Phase Microextraction } \\
\hline Beef & Heterocyclic amines & In-tube SPME & [63] \\
\hline Fatty foods & Endocrine disruptors & In-tube SPME & [64] \\
\hline Baby food & Furan & Headspace-SPME & [58] \\
\hline Fish & Formaldehyde & Headspace-SPME & [59] \\
\hline Beer & Volatiles & Headspace-SPME & [50] \\
\hline Cow's milk & Phthalate esters & Headspace-SPME & [73] \\
\hline Cheese & Volatiles & Headspace-SPME & [74] \\
\hline Food & Volatiles & Headspace-SPDE & [75] \\
\hline Honey & Amitraz & Headspace-SPDE & [76] \\
\hline Wines & Off-flavors & Headspace-SPDE & [77] \\
\hline \multicolumn{4}{|c|}{ Stir Bar Sorptive Extraction } \\
\hline Coffee & Aroma profiles & SBSE, HSSE & [51] \\
\hline Grape juice & Volatiles & SBSE, SDE & [78] \\
\hline Sugarcane juice & Pesticides and benzo $[a]$ pyrene & SBSE & [79] \\
\hline Honey & Pesticides & SBSE & [80] \\
\hline Alcoholic beverages & Flavor profile & SBSE, SPME & {$[52]$} \\
\hline Oranges & Pesticides & SBSE & [67] \\
\hline Plants & Aroma profile & HSSE & [69] \\
\hline Food & Aroma profile & HSSE & [70] \\
\hline
\end{tabular}

Note: SDME, single-drop microextraction; HF-LPME, hollow fiber protected liquid-phase microextraction; RAM, restricted access media; MIPs, molecularly imprinted polymers; HSSE, headspace sorptive extraction; SDE, steam distillation extraction. 


\section{REFERENCES}

1. Liu, H.H. and Dasgupta, P.K. Analytical chemistry in a drop. Solvent extraction in a microdrop. Analytical Chemistry, 1996, 68(11), 1817-1821.

2. Wood, D.C., Miller, J.M., and Christ, I. Headspace liquid microextraction. LCGC Europe, 2004, November, 573-579.

3. Psillakis, E. and Kalogerakis, N. Developments in liquid-phase microextraction. TrAC, Trends in Analytical Chemistry, 2003, 22(9), 565-574.

4. Zhao, E., Han, L., Jiang, S., Wang, Q., and Zhou, Z. Application of a single-drop microextraction for the analysis of organophosphorus pesticides in juice. Journal of Chromatography A, 2006, 1114(2), 269-273.

5. Rasmussen, K.E. and Pedersen-Bjergaard, S. Developments in hollow fibre-based, liquid-phase microextraction. TrAC, Trends in Analytical Chemistry, 2004, 23(1), 1-10.

6. Gonzalez-Penas, E., Leache, C., Viscarret, M., Perez de Obanos, A., Araguas, C., and Lopez de Cerain, A. Determination of ochratoxin $\mathrm{A}$ in wine using liquid-phase microextraction combined with liquid chromatography with fluorescence detection. Journal of Chromatography A, 2004, 1025(2), 163-168.

7. Bjorhovde, A., Halvorsen, T.G., Rasmussen, K.E., and Pedersen-Bjergaard, S. Liquid-phase microextraction of drugs from human breast milk. Analytica Chimica Acta, 2003, 491(2), 155-161.

8. Zhu, L., Huey Ee, K., Zhao, L., and Lee, H.K. Analysis of phenoxy herbicides in bovine milk by means of liquid-liquid-liquid microextraction with a hollow-fiber membrane. Journal of Chromatography A, 2002, 963(1-2), 335-343.

9. Michulec, M. and Wardencki, W. The application of single drop extraction technique for chromatographic determination of solvent residues in edible oils and pharmaceutical products. Chromatographia, 2006, 64(3), 191-197.

10. Hennion, M.C. Solid-phase extraction: Method development, sorbents, and coupling with liquid chromatography. Journal of Chromatography A, 1999, 856(1-2), 3-54.

11. Thurman, E.M. and Mills, M.S. Solid Phase Extraction: Principles and Practice. 1998. Chemical Analysis: A Series of Monographs on Analytical Chemistry and Its Applications. John Wiley and Sons Inc., New York.

12. Fritz, J.S. Analytical Solid-Phase Extraction. 1999. Wiley-VCH, New York.

13. Poole, C.F., Gunatilleka, A.D., and Sethuraman, R. Contributions of theory to method development in solid-phase extraction. Journal of Chromatography A, 2000, 885(1-2), 17-39.

14. Mitra, S. (Ed.). Sample Preparation Techniques in Analytical Chemistry. 2003, Vol. 162. Wiley-Interscience, Hoboken, NJ.

15. Fontanals, N., Marce, R.M., and Borrull, F. New hydrophilic materials for solid-phase extraction. TrAC, Trends in Analytical Chemistry, 2005, 24(5), 394-406.

16. Wang, J.X., Jiang, D.Q., Gu, Z.Y., and Yan, X.P. Multiwalled carbon nanotubes coated fibers for solidphase microextraction of polybrominated diphenyl ethers in water and milk samples before gas chromatography with electron-capture detection. Journal of Chromatography A, 2006, 1137(1), 8-14.

17. Buldini, P.L., Ricci, L., and Sharma, J.L. Recent applications of sample preparation techniques in food analysis. Journal of Chromatography A, 2002, 975(1), 47-70.

18. Breithaupt, D.E. Determination of folic acid by ion-pair RP-HPLC in vitamin-fortified fruit juices after solid-phase extraction. Food Chemistry, 2001, 74(4), 521-525.

19. Gonzalez, M., Gallego, M., and Valcarcel, M. Gas chromatographic flow method for the preconcentration and simultaneous determination of antioxidant and preservative additives in fatty foods. Journal of Chromatography A, 1999, 848(1-2), 529-536.

20. Molins-Legua, C. and Campins, F. Solid phase extraction of amines. Analytica Chimica Acta, 2005, 546(2), 206-220.

21. Li, H., Kijak, P.J., Turnipseed, S.B., and Cui, W. Analysis of veterinary drug residues in shrimp: A multiclass method by liquid chromatography-quadrupole ion trap mass spectrometry. Journal of Chromatography $B, 2006,836(1-2), 22-38$.

22. Peng, L., Farkas, T., Loo, L., Dixon, A., Teuscher, J., and Kallury, K. Rapid and Reproducible Extraction of Acrylamide in French Fries Using a Single SPE Sorbent-Strata-X-C. Phenomenex Applications Note TN-007. 2007. Phenomenex, Inc., Torrance, CA. 
23. Toribio, F., Moyano, E., Puignou, L., and Galceran, M.T. Comparison of different commercial solid-phase extraction cartridges used to extract heterocyclic amines from a lyophilised meat extract. Journal of Chromatography A, 2000, 880(1-2), 101-112.

24. Desilets, C.P., Rounds, M.A., and Regnier, F.E. Semipermeable-surface reversed-phase media for highperformance liquid chromatography. Journal of Chromatography A, 1991, 544, 25-39.

25. Souverain, S., Rudaz, S., and Veuthey, J.-L. Restricted access materials and large particle supports for on-line sample preparation: An attractive approach for biological fluids analysis. Journal of Chromatography $B, 2004,801(2), 141-156$.

26. Blahova, E., Bovanova, L., and Brandsteterova, E. Direct HPLC analysis of trimethoprim in milk. Journal of Liquid Chromatography and Related Technologies, 2001, 24(19), 3027-3035.

27. Heinig, K. and Bucheli, F. Application of column-switching liquid chromatography-tandem mass spectrometry for the determination of pharmaceutical compounds in tissue samples. Journal of Chromatography B: Analytical Technologies in the Biomedical and Life Sciences, 2002, 769(1), 9-26.

28. Hennion, M.C. and Pichon, V. Immuno-based sample preparation for trace analysis. Journal of Chromatography A, 2003, 1000(1-2), 29-52.

29. Watanabe, E., Yoshimura, Y., Yuasa, Y., and Nakazawa, H. Immunoaffinity column clean-up for the determination of imazalil in citrus fruits. Analytica Chimica Acta, 2001, 433(2), 199-206.

30. Pichon, V., Krasnova, A.I., and Hennion, M.C. Development and characterization of an immunoaffinity solid-phase-extraction sorbent for trace analysis of propanil and related phenylurea herbicides in environmental waters and in beverages. Chromatographia, 2004, 60(Suppl.), S221-S226.

31. Gilbert, J. and Anklam, E. Validation of analytical methods for determining mycotoxins in foodstuffs. TrAC, Trends in Analytical Chemistry, 2002, 21(6-7), 468-486.

32. Holtzapple, C.K., Buckley, S.A., and Stanker, L.H. Immunosorbents coupled on-line with liquid chromatography for the determination of fluoroquinolones in chicken liver. Journal of Agricultural and Food Chemistry, 1999, 47(7), 2963-2968.

33. Stolker, A.A.M., Schwillens, P.L.W.J., van Ginkel, L.A., and Brinkman, U.A.T. Comparison of different liquid chromatography methods for the determination of corticosteroids in biological matrices. Journal of Chromatography A, 2000, 893(1), 55-67.

34. Lanza, F. and Sellergren, B. The application of molecular imprinting technology to solid phase extraction. Chromatographia, 2001, 53(11-12), 599-611.

35. Caro, E., Marce, R.M., Cormack, P.A.G., Sherrington, D.C., and Borrull, F. On-line solid-phase extraction with molecularly imprinted polymers to selectively extract substituted 4-chlorophenols and 4-nitrophenol from water. Journal of Chromatography A, 2003, 995(1-2), 233-238.

36. Stevenson, D. Immuno-affinity solid-phase extraction. Journal of Chromatography B: Biomedical Sciences and Applications, 2000, 745(1), 39-48.

37. Andersson, L.I. Molecular imprinting for drug bioanalysis: A review on the application of imprinted polymers to solid-phase extraction and binding assay. Journal of Chromatography B: Biomedical Sciences and Applications, 2000, 739(1), 163-173.

38. Andersson, L.I. Molecular imprinting: Developments and applications in the analytical chemistry field. Journal of Chromatography B: Biomedical Sciences and Applications, 2000, 745(1), 3-13.

39. Muldoon, M.T. and Stanker, L.H. Molecularly imprinted solid phase extraction of atrazine from beef liver extracts. Analytical Chemistry, 1997, 69(5), 803-808.

40. Zander, A., Findlay, P., Renner, T., Sellergren, B., and Swietlow, A. Analysis of nicotine and its oxidation products in nicotine chewing gum by a molecularly imprinted solid-phase extraction. Analytical Chemistry, 1998, 70(15), 3304-3314.

41. Puoci, F., Garreffa, C., Iemma, F., Muzzalupo, R., Spizzirri, U.G., and Picci, N. Molecularly imprinted solid phase extraction for detection of Sudan I in food matrices. Food Chemistry, 2005, 93(2), 349-353.

42. Arthur, C.L. and Pawliszyn, J. Solid phase microextraction with thermal desorption using fused silica optical fibers. Analytical Chemistry, 1990, 62, 2145-2148.

43. Janusz, P. Solid Phase Microextraction: Theory and Practice. 1997. Wiley, New York.

44. Wercinski, S.A.S. Solid Phase Microextraction: A Practical Guide. 1999. Marcel Dekker, New York.

45. Pawliszyn, J. Applications of Solid Phase Microextraction (RSC Chromatography Monographs). 1999, 1st ed. Royal Society of Chemistry, Cambridge, United Kingdom. 
46. Kataoka, H., Lord, H.L., and Pawliszyn, J. Applications of solid-phase microextraction in food analysis. Journal of Chromatography A, 2000, 880(1-2), 35-62.

47. Falch, I. (Ed.). SPME-metal fibre assemblies. The Reporter (Europe), 2006, 22 Sigma Aldrich, 12.

48. Miralles-Garcia, J., Ducki, S., and Storey, D.M. 2005. ARF05 presentation. Plymouth, United Kingdom.

49. Liu, M., Zeng, Z., and Xiong, B. Preparation of novel solid-phase microextraction fibers by sol-gel technology for headspace solid-phase microextraction-gas chromatographic analysis of aroma compounds in beer. Journal of Chromatography A, 2005, 1065(2), 287-299.

50. Bicchi, C., Iori, C., Rubiolo, P., and Sandra, P. Headspace sorptive extraction (HSSE), stir bar sorptive extraction (SBSE), and solid phase microextraction (SPME) applied to the analysis of roasted arabica coffee and coffee brew. Journal of Agricultural and Food Chemistry, 2002, 50(3), 449-459.

51. Demyttenaere, J.C.R., Sanchez Martinez, J.I., Verhe, R., Sandra, P., and De Kimpe, N. Analysis of volatiles of malt whisky by solid-phase microextraction and stir bar sorptive extraction. Journal of Chromatography A, 2003, 985(1-2), 221-232.

52. Bicchi, C., Cordero, C., and Rubiolo, P. A survey on high-concentration-capability headspace sampling techniques in the analysis of flavors and fragrances. Journal of Chromatographic Science, 2004, 42(8), 402-409.

53. Wilkes, J.G., Conte, E.D., Kim, Y., Holcomb, M., Sutherland, J.B., and Miller, D.W. Sample preparation for the analysis of flavors and off-flavors in foods. Journal of Chromatography A, 2000, 880(1-2), 3-33.

54. Zhang, Z.Y., Poerschmann, J., and Pawliszyn, J. Direct solid phase microextraction of complex aqueous samples with hollow fibre membrane protection. Analytical Communications, 1996, 33(7), 219-221.

55. Stashenko, E.E. and Martinez, J.R. Derivatization and solid-phase microextraction. TrAC, Trends in Analytical Chemistry, 2004, 23(8), 553-561.

56. Wardencki, W., Michulec, M., and Curylo, J. A review of theoretical and practical aspects of solid-phase microextraction in food analysis. International Journal of Food Science and Technology, 2004, 39(7), 703-717.

57. Bianchi, F., Careri, M., Mangia, A., and Musci, M. Development and validation of a solid phase microextraction-gas chromatography-mass spectrometry method for the determination of furan in baby-food. Journal of Chromatography A, 2006, 1102(1-2), 268-272.

58. Bianchi, F., Careri, M., Musci, M., and Mangia, A. Fish and food safety: Determination of formaldehyde in 12 fish species by SPME extraction and GC-MS analysis. Food Chemistry, 2007, 100(3), $1049-1053$.

59. Lord, H. and Pawliszyn, J. Microextraction of drugs. Journal of Chromatography A, 2000, 902(1), 17-63.

60. Lipinski, J. Automated solid phase dynamic extraction-Extraction of organics using a wall coated syringe needle. Fresenius Journal of Analytical Chemistry, 2001, 369(1), 57-62.

61. Kataoka, H. Automated sample preparation using in-tube solid-phase microextraction and its application-A review. Analytical and Bioanalytical Chemistry, 2002, 373(1-2), 31-45.

62. Kataoka, H. and Pawliszyn, J. Development of in-tube solid-phase microextraction/liquid chromatography/electrospray ionization mass spectrometry for the analysis of mutagenic heterocyclic amines. Chromatographia, 1999, 50(9-10), 532-538.

63. Kataoka, H., Ise, M., and Narimatsu, S. Automated on-line in-tube solid-phase microextraction coupled with high performance liquid chromatography for the analysis of bisphenol A, alkylphenols, and phthalate esters in food contacted with plastics. Journal of Separation Science, 2002, 25(1-2), 77-85.

64. Baltussen, E., Sandra, P., David, F., and Cramers, C. Stir bar sorptive extraction (SBSE), a novel extraction technique for aqueous samples: Theory and principles. Journal of Microcolumn Separations, 1999, 11(10), 737-747.

65. Bicchi, C., Cordero, C., Liberto, E., Rubiolo, P., Sgorbini, B., David, F., and Sandra, P. Dual-phase twisters: A new approach to headspace sorptive extraction and stir bar sorptive extraction. Journal of Chromatography A, 2005, 1094(1-2), 9-16.

66. Blasco, C., Font, G., and Pico, Y. Comparison of microextraction procedures to determine pesticides in oranges by liquid chromatography-mass spectrometry. Journal of Chromatography A, 2002, 970(1-2), 201-212.

67. Sandra, P., Tienpont, B., Vercammen, J., Tredoux, A., Sandra, T., and David, F. Stir bar sorptive extraction applied to the determination of dicarboximide fungicides in wine. Journal of Chromatography A, 2001, 928(1), 117-126. 
68. Bicchi, C., Cordero, C., Iori, C., Rubiolo, P., and Sandra, P. Headspace sorptive extraction (HSSE) in the headspace analysis of aromatic and medicinal plants. HRC Journal of High Resolution Chromatography, 2000, 23(9), 539-546.

69. Tienpont, B., Sandra, P., David, F., and Bicchi, C. High capacity headspace sorptive extraction. Journal of Microcolumn Separations, 2000, 12(11), 577-584.

70 Posyniak, A., Zmudzki, J., and Semeniuk, S. Effects of the matrix and sample preparation on the determination of fluoroquinolone residues in animal tissues. Journal of Chromatography A, 2001, 914, 89-94.

71. Tankeviciute, A., Kazlauskas, R., and Vickackaite, V. Headspace extraction of alcohols into a single drop. Analyst, 2001, 126(10), 1674-1677.

72. Feng, Y.L., Zhu, J., and Sensenstein, R. Development of a headspace solid-phase microextraction method combined with gas chromatography mass spectrometry for the determination of phthalate esters in cow milk. Analytica Chimica Acta, 2005, 538(1-2), 41-48.

73. Mallia, S., Fernandez-Garcia, E., and Olivier Bosset, J. Comparison of purge and trap and solid phase microextraction techniques for studying the volatile aroma compounds of three European PDO hard cheeses. International Dairy Journal, 2005, 15(6-9), 741-758.

74. Bicchi, C., Cordero, C., Liberto, E., Rubiolo, P., and Sgorbini, B. Automated headspace solid-phase dynamic extraction to analyse the volatile fraction of food matrices. Journal of Chromatography A, 2004, 1024(1-2), 217-226.

75. Hahn, H., Nothhelfer, A., and Preuss, S. Determination of amitraz in honey by SPDE-GC-MS/MS. 2003. Chromtech Application Note 301. Chromtech, Idstein, Germany.

76. Chokshi, K. and Christ, I. Comparative SPDE and SPME Studies for Analysis of Off-flavors in Wines. 2006. Chromsys LLC, Alexandria, VA. http://www.chromsys.com/Applications/wine.htm.

77. Caven-Quantrill, D.J. and Buglass, A.J. Comparison of micro-scale simultaneous distillation-extraction and stir bar sorptive extraction for the determination of volatile organic constituents of grape juice. Journal of Chromatography A, 2006, 1117(2), 121-131.

78. Zuin, V.G., Schellin, M., Montero, L., Yariwake, J.H., Augusto, F., and Popp, P. Comparison of stir bar sorptive extraction and membrane-assisted solvent extraction as enrichment techniques for the determination of pesticide and benzo[a]pyrene residues in Brazilian sugarcane juice. Journal of Chromatography A, 2006, 1114(2), 180-187.

79. Blasco, C., Fernandez, M., Pico, Y., and Font, G. Comparison of solid-phase microextraction and stir bar sorptive extraction for determining six organophosphorus insecticides in honey by liquid chromatographymass spectrometry. Journal of Chromatography A, 2004, 1030(1-2), 77-85. 



\title{
3 Supercritical Fluid Extraction in Food Analysis
}

\author{
Ruhan Askin, Motonobu Goto, and Mitsuru Sasaki
}

\section{CONTENTS}

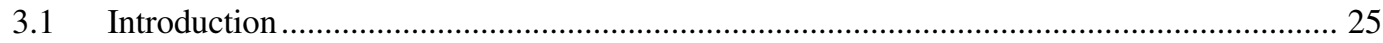

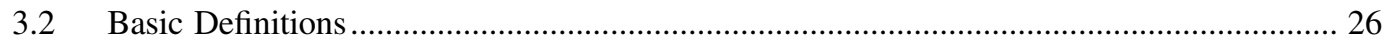



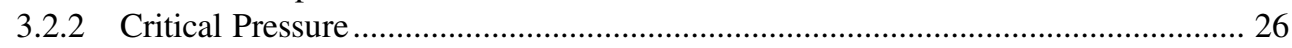

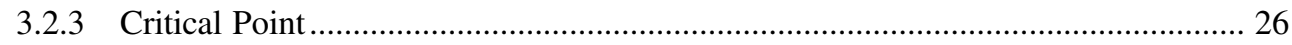

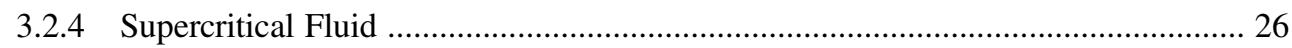

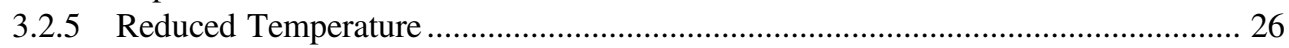

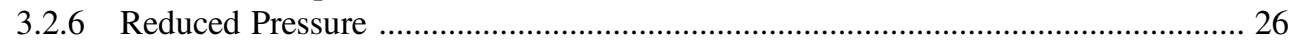

3.2.7 Supercritical Fluid Extraction ......................................................................... 27

3.2.8 Coupled Supercritical Fluid Extraction-Supercritical Fluid Chromatography ....... 27

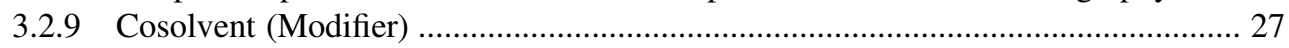

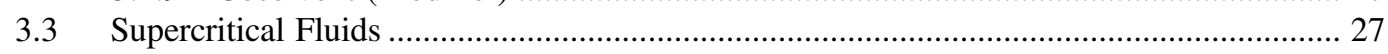

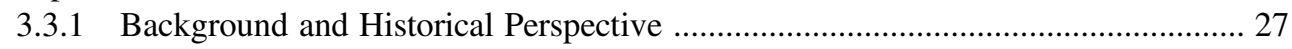

3.3.2 Basic Properties and Fundamentals of Supercritical Fluids ................................... 27

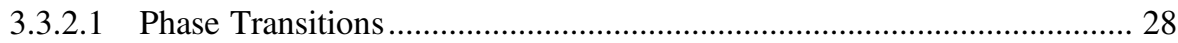

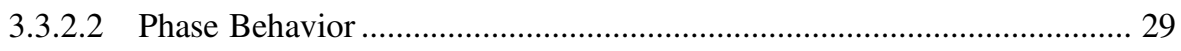

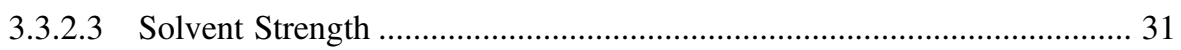

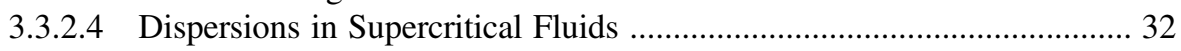

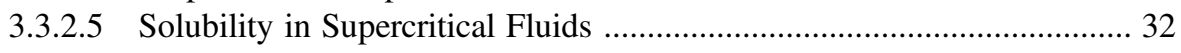



3.4 Supercritical Fluid Extraction Mechanism ............................................................... 36

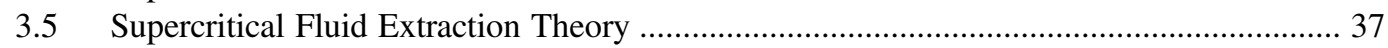

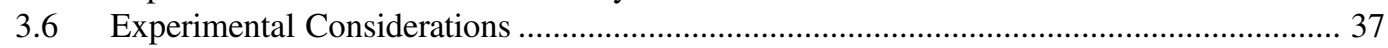

3.7 Applications and Commercial Processes of Supercritical Fluids ..................................... 39

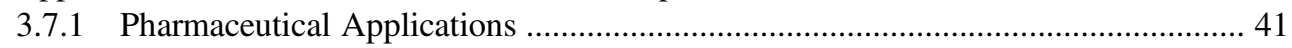

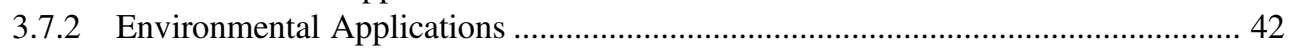

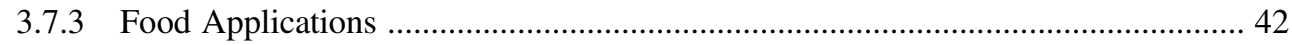

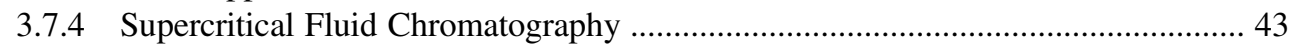



3.9 Current Trends and Future Expects of Supercritical Fluids ........................................... 51

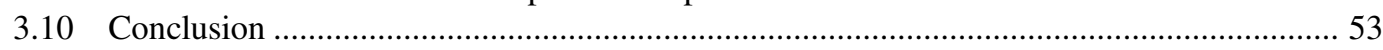

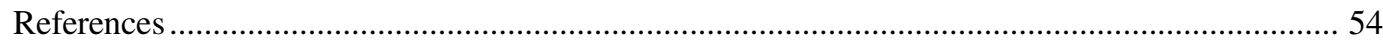

\subsection{INTRODUCTION}

This chapter is an overview of the current state of the science and technology of supercritical fluids. The principal objective is to acquaint the reader with the unusual properties of supercritical fluids, and 
with the ways some basic principles are essential in understanding the supercritical fluid extraction (SFE) technique and the independence of relevant process parameters that are exploited for a variety of applications in cases of both SFE and supercritical fluid chromatography (SFC) in the food industry. The unusual solvent properties of supercritical fluids, together with their thermodynamic behavior near a critical point, are explained within the framework of fluid-phase diagrams. Characterizing the behavior of supercritical fluids still offers many challenges to scientists. Engineers have exploited the peculiarities of supercritical fluids to great advantage to design new instruments and processes. These proceedings are an example of the dialogue between scientists and engineers that is needed to deepen the understanding of this interesting medium and to widen the field of applications.

The basic philosophy of utilization is centered on the fact that the properties of supercritical fluids can be varied from gas-like to liquid-like values by simply adjusting the pressure. These fluids are therefore very attractive as tunable process solvents or reaction media.

To summarize, in this chapter the basic knowledge and terminology required for understanding supercritical fluid applications including SFE together with SFC are introduced at an elementary level.

\subsection{BASIC DEFINITIONS}

\subsubsection{Critical Temperature}

The critical temperature $\left(T_{\mathrm{c}}\right)$ is the maximum temperature at the critical point at which a gas can be converted into a liquid by an increase in pressure.

\subsubsection{Critical Pressure}

The critical pressure $\left(P_{\mathrm{c}}\right)$ is the minimum pressure that would suffice to liquefy a substance at its critical temperature. Above the critical pressure, increasing the temperature will not cause a fluid to vaporize to give a two-phase system.

\subsubsection{Critical Point}

The characteristic temperature $\left(T_{\mathrm{c}}\right)$ and pressure $\left(P_{\mathrm{c}}\right)$ above which a gas cannot be liquefied.

\subsubsection{Supercritical Fluid}

The defined state of a compound, mixture, or element above its critical pressure $\left(P_{\mathrm{c}}\right)$ and critical temperature $\left(T_{\mathrm{c}}\right)$. It is a gas-like, compressible fluid that takes a shape of its container and fills it. It is not a liquid but has liquid-like densities $(0.1-1 \mathrm{~g} / \mathrm{mL})$ and solvating power.

\subsubsection{Reduced Temperature}

The reduced temperature $\left(T_{\mathrm{r}}\right)$ is the ratio of the temperature $(T)$ in the system to the critical temperature $\left(T_{\mathrm{c}}\right)$.

$$
T_{\mathrm{r}}=T / T_{\mathrm{c}}
$$

\subsubsection{Reduced Pressure}

The reduced pressure $\left(P_{\mathrm{r}}\right)$ is the ratio of the pressure in the system $(P)$ to the critical pressure $\left(P_{\mathrm{c}}\right)$.

$$
P_{\mathrm{r}}=P / P_{\mathrm{c}}
$$




\subsubsection{Supercritical Fluid Extraction}

Extraction of a material using a supercritical fluid. The extracted material is usually recovered by reducing the pressure or increasing the temperature of the extraction fluid and allowing the volatile components of the mobile phase to evaporate. Instrumentally, supercritical fluid extraction can use many of the components of a supercritical fluid chromatographic system. It can be used either as an online sample introduction method for a chromatographic separation or as an offline sample preparation method.

\subsubsection{Coupled Supercritical Fluid Extraction-Supercritical Fluid Chromatography}

In this system a sample is extracted with a supercritical fluid, which then places the extracted material in the inlet port of a supercritical fluid chromatographic system. The extract is then chromatographed directly using a supercritical fluid.

\subsubsection{Cosolvent (Modifier)}

Organic solvents that are used in small quantities in many SFE procedures have become apparent as the technique has matured. These cosolvents are generally used to increase the solubility of the analyte or possibly to increase the separation of co-extractives. Cosolvents such as ethanol have been used to increase the solubility of phospholipids in supercritical carbon dioxide $\left(\mathrm{SCCO}_{2}\right)$ [1,2]. Performing SFE with cosolvents usually results in a higher weight percent of fat over that recorded with pure $\mathrm{CO}_{2}$.

\subsection{SUPERCRITICAL FLUIDS}

\subsubsection{Background and Historical Perspective}

In 1822, Baron Charles Cagniard de la Tour discovered the critical point of a substance in his famous cannon barrel experiments. Listening to discontinuities in the sound of a rolling flint ball in a sealed cannon filled with fluids at various temperatures, he observed the critical temperature. Above this temperature, the densities of the liquid and gas phases become equal and the distinction between them disappears, resulting in a single supercritical fluid phase. Although their unique solvent properties were first reported over 100 years ago, only about 20 years ago did supercritical fluids enter the contemporary technical and industrial scene, with the simultaneous appearance in chemical and engineering journals of reports about applications in decaffeinating coffee and tea, extracting hops flavors used in brewing, and extracting aromas and flavors from spices and herbs; by the early 1980s several huge plants (tens to hundreds of millions of pounds per year) were operating in Europe, United States, and Japan. In the intervening years, supercritical fluids have been applied in the development of new or improved products achieving specifications that cannot be met by other industrial processing methods. In Table 3.1, the critical properties are shown for some components, which are commonly used as supercritical fluids.

Supercritical fluid extraction utilizes the ability of certain chemicals to become excellent solvents for certain solutes under a combination of temperature and pressure [3,4]. The term supercritical fluid describes a gas or liquid at conditions above its critical temperature and pressure, i.e., above the critical point.

\subsubsection{Basic Properties and Fundamentals of Supercritical Fluids}

Two researchers, Hannay and Hogarth, at a meeting of the Royal Society (London) in 1879, reported that supercritical fluids have a pressure-dependent dissolving power-the higher the pressure, the higher their dissolving power [5]. They described their work and summarized their findings as follows: "We have the phenomenon of a solid dissolving in a gas, and when the solid is precipitated by reducing the pressure, it is brought down as a 'snow' in the gas." The researchers 


\section{TABLE 3.1}

\section{Critical Properties of Various Solvents}

\begin{tabular}{|c|c|c|c|c|c|}
\hline \multirow[b]{2}{*}{ Solvent } & \multirow{2}{*}{$\begin{array}{l}\text { Molecular Weight } \\
\qquad(\mathrm{g} / \mathrm{mol})\end{array}$} & \multirow{2}{*}{$\begin{array}{c}\text { Temperature } \\
\text { (K) }\end{array}$} & \multicolumn{2}{|c|}{ Pressure } & \multirow{2}{*}{$\begin{array}{l}\text { Density } \\
\left(\mathrm{g} / \mathrm{cm}^{3}\right)\end{array}$} \\
\hline & & & $\overline{(\mathrm{MPa})}$ & (bar) & \\
\hline Carbon dioxide & 44.01 & 304.1 & 7.38 & 73.8 & 0.469 \\
\hline Water & 18.02 & 647.3 & 22.12 & 221.2 & 0.348 \\
\hline Methane & 16.04 & 190.4 & 4.60 & 46.0 & 0.162 \\
\hline Ethane & 30.07 & 305.3 & 4.87 & 48.7 & 0.203 \\
\hline Propane & 44.09 & 369.8 & 4.25 & 42.5 & 0.217 \\
\hline Ethylene & 28.05 & 282.4 & 5.04 & 50.4 & 0.215 \\
\hline Propylene & 42.08 & 364.9 & 4.60 & 46.0 & 0.232 \\
\hline Methanol & 32.04 & 512.6 & 8.09 & 80.9 & 0.272 \\
\hline Ethanol & 46.07 & 513.9 & 6.14 & 61.4 & 0.276 \\
\hline Acetone & 58.08 & 508.1 & 4.70 & 47.0 & 0.278 \\
\hline
\end{tabular}

referred to supercritical fluids as gases, which, in fact, they are. In the interest of brevity, the term "gas," or the abbreviation "SCF" for supercritical fluids, will be used liberally throughout this chapter. The solubility behavior was not exploited until many, many years later, but it is of historical interest to relate some of the events surrounding their findings. There arose serious (but, as were the times, polite) controversy at the October 1879 society meeting. Some of the members who were present said, "Gases cannot dissolve solid compounds. The researchers must have erred and instead found solubility in superheated liquids." In other carefully planned and executed experiments, the researchers did, however, substantiate their previous findings. Gases, in other words, SCF, could indeed dissolve many compounds.

\subsubsection{Phase Transitions}

Figure 3.1 shows isotherms and typical behavior of a real gas as it is subjected to different pressures and temperatures. It should be noted that there are no phase transitions above $T_{\mathrm{c}}$. The isotherms

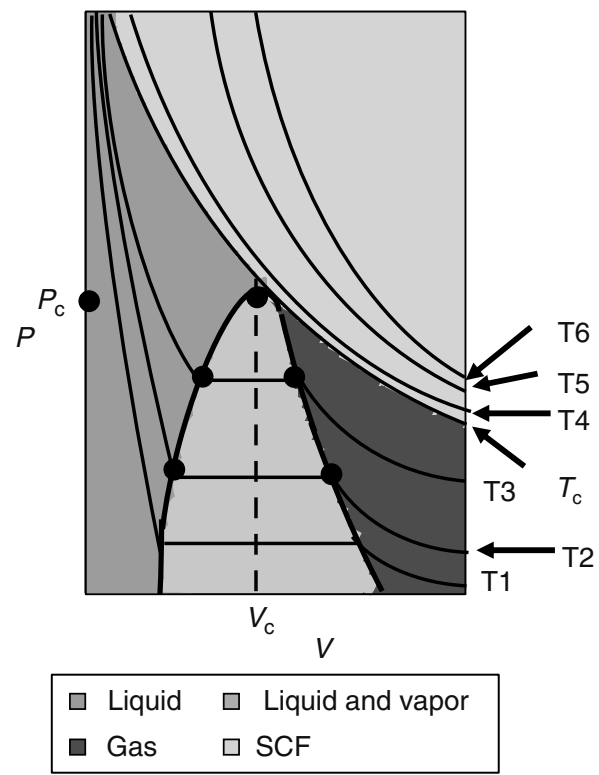

FIGURE 3.1 (See color insert following page 240.) Phase diagram for a typical real gas. 


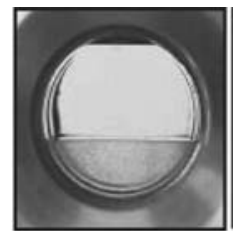

(1)

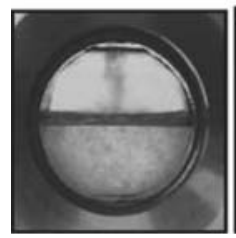

(5)

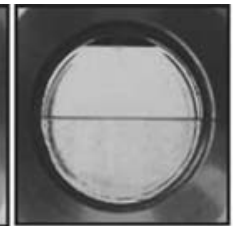

(2)

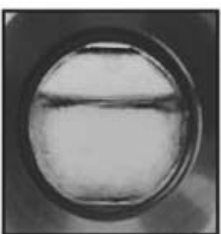

(6)

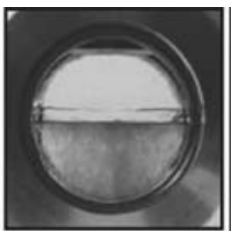

(3)

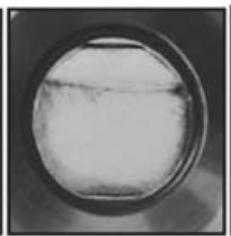

(7)

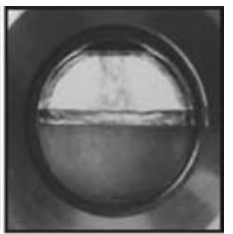

(4)

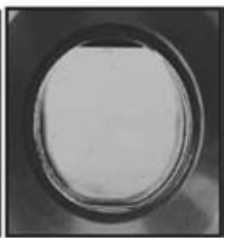

(8)

FIGURE 3.2 Change from two definite phases to one supercritical phase.

shown in the figure are smooth; they have no tie lines. Tie lines are the horizontal portions of the isotherms, though these are really not really part of the isotherms.

In addition, Figure 3.2 shows three photos of the same system. From left to right, the temperature is increasing. In the upper-left photo, there are two phases present, liquid and gas, and the distinction between them is obvious. The following are near the critical temperature, so the separation of the two phases is becoming obscured. In the photo on the bottom-right, there is no phase distinction, so this is above the critical temperature and is a supercritical fluid as it is also shown in Figure 3.3.

\subsubsection{Phase Behavior}

The observations can be explained by looking at the phase diagram of a pure component, e.g., carbon dioxide. Carbon dioxide was substituted for organic solvents (hexane, benzene, carbon tetrachloride, methylene chloride, methanol, and acetone) used in conventional extraction methods. $\mathrm{CO}_{2}$ is probably the most studied SCF as it is nonflammable, harmless, noncorrosive, inexpensive, and nontoxic, and it can be obtained with high purity [6]. In the case of carbon dioxide, the critical

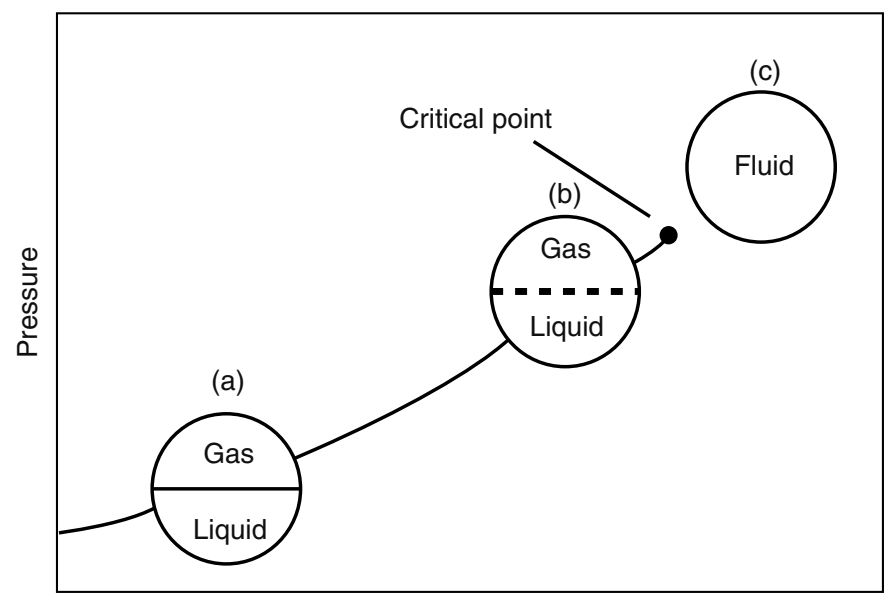

Temperature

FIGURE 3.3 Disappearance of the meniscus at the critical point. 


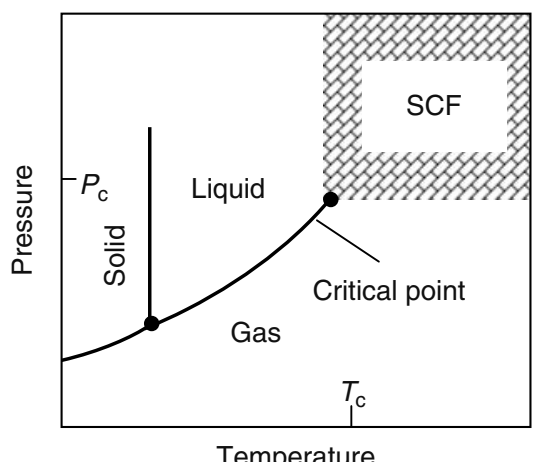

FIGURE 3.4 Pressure-temperature phase diagram.

point is at $304.06 \mathrm{~K}$ and $7.386 \mathrm{MPa} . \mathrm{CO}_{2}$ is the solvent of choice for use in SFE because it is "GRAS"nonflammable, noncorrosive, and inexpensive. In addition, $\mathrm{CO}_{2}$ has a low critical temperature, which can help prevent thermal degradation of food components when they are being extracted. In Figures 3.4 and 3.5, two projections of the phase diagram of carbon dioxide are shown. Drawing from physical chemistry texts, the critical point is located at the end of the vapor pressure curve, and Figure 3.4 shows a generalized vapor pressure curve and its end. The accented region in the figure denotes the supercritical fluid space where many gases exhibit the propensity to dissolve materials.

In the pressure-temperature phase diagram, the boiling line, which separates the vapor and liquid region and ends in the critical point, is observed. At the critical point, the densities of the equilibrium liquid-phase and the saturated vapor-phases become equal, resulting in the formation of a single supercritical phase. This can be observed in the density-pressure phase diagram for carbon dioxide, as shown in Figure 3.5, where the critical point is located at $304.1 \mathrm{~K}$ and $7.38 \mathrm{MPa}$ (73.8 bar). With increasing temperatures, the liquid-vapor density gap decreases, up to the critical

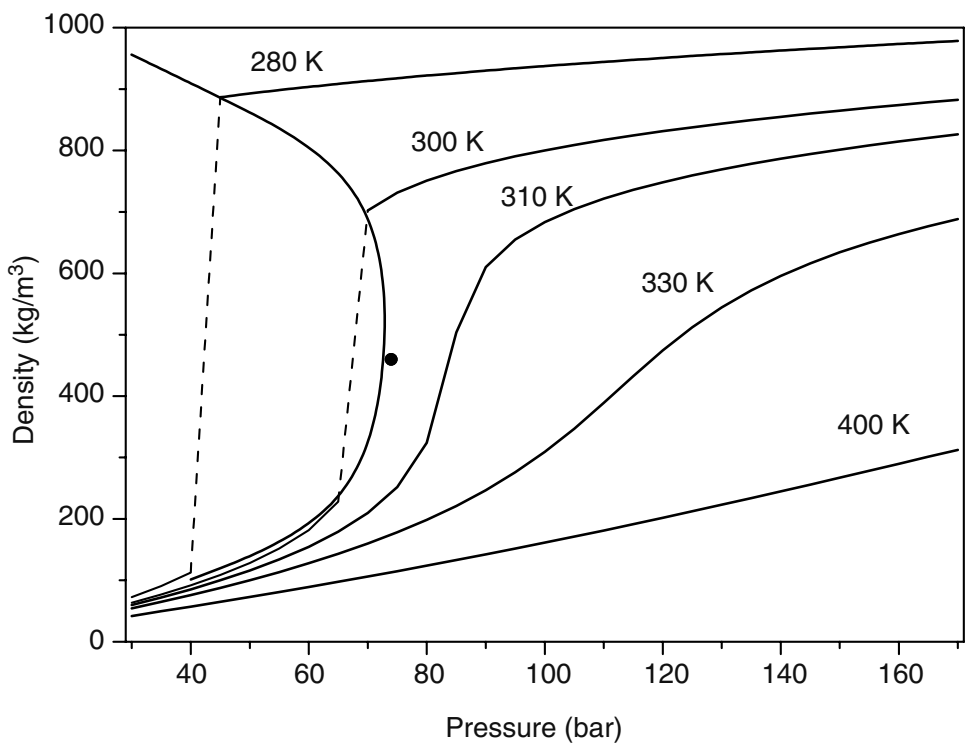

FIGURE 3.5 Carbon dioxide density-pressure phase diagram. 


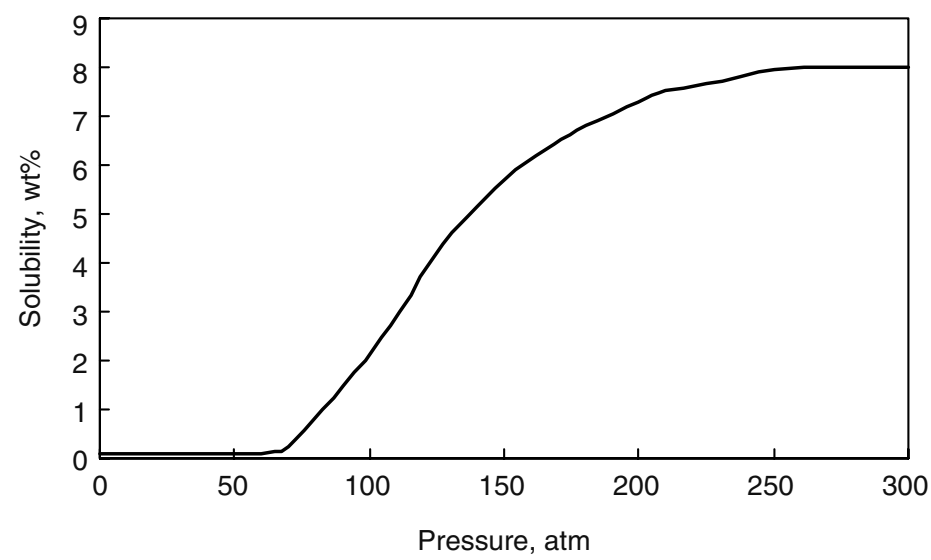

FIGURE 3.6 Solubility of naphthalene in supercritical carbon dioxide $\left(45^{\circ} \mathrm{C}\right)$.

temperature, at which the discontinuity disappears. Thus, above the critical temperature, a gas cannot be liquefied by pressure.

By definition, a supercritical fluid is a substance above both its critical temperature and pressure. In a practical sense, the area of interest in supercritical fluids for processing and separation purposes is limited to temperatures in the vicinity of the critical point, where large gradients in the physical properties are observed. The changes near the critical point are not limited to density. Many other physical properties also show large gradients with pressure near the critical point, e.g., viscosity, the relative permittivity, and the solvent strength, which are all closely related to the density. At higher temperatures, the fluid starts to behave like a gas, as can be seen in Figure 3.5. For carbon dioxide at $400 \mathrm{~K}$, the density increases almost linearly with pressure [3].

In a very brief explanation of the technology, supercritical fluids exhibit a pressure-dependent dissolving power, the higher the pressure, the higher the dissolving power, and this property can be applied to purification, extraction, fractionation, and recrystallization of a wide host of materials. Being related to such important properties, pressure-dependent dissolving power is illustrated in Figure 3.6, which shows the solubility of a much-studied model compound, naphthalene, in $\mathrm{SCCO}_{2}$. At pressure levels less than the critical pressure of $\mathrm{CO}_{2}$, the solubility of naphthalene is essentially nil, but as the pressure is raised, the solubility increases to quite high levels.

Naphthalene solubility has been studied by at least a dozen groups in a variety of gases, and for an interesting historical aside, Büchner, of Nobel Prize fame, was the first person to study the solubility of naphthalene in $\mathrm{SCCO}_{2}$ [7]. The Proceedings of the Royal Society (and other journals) describes much of the work during the early years of supercritical fluids activity, and naphthalene is still studied today for the information its solubility behavior presents to new researchers in the SCF field [8].

\subsubsection{Solvent Strength}

The density of a supercritical fluid is extremely sensitive to minor changes in temperature and pressure near the critical point. The density of fluids is closer to that of organic liquids but the solubility of solids can be 3-10 orders of magnitude higher. The enhancement of solubilities was discovered in 1870s for the potassium iodide-ethanol system. The solvent strength of a fluid can be expressed by the solubility parameter, $d$, which is the square root of the cohesive energy density and is defined rigorously from first principles. A plot of the solubility parameter for carbon dioxide versus pressure would resemble a plot of density versus pressure. This confirms that the solvation strength of a supercritical fluid is directly related to the fluid density. Thus the solubility of a solid can be manipulated by making slight changes in temperatures and pressures. 
Another attractive feature of supercritical fluids is that the properties lie between that of gases and liquids. A supercritical fluid has densities similar to that of liquids, while the viscosities and diffusivities are closer to that of gases. Thus, a supercritical fluid can diffuse faster in a solid matrix than a liquid, yet possess a solvent strength to extract the solute from the solid matrix [9].

\subsubsection{Dispersions in Supercritical Fluids}

The ability to design surfactants for the interface between water (or organics) and supercritical fluids offers new avenues in protein and polymer chemistry, separation science, reaction engineering, waste minimization, and treatment. Surfactant design, which is reasonably well understood for conventional reverse micelles and water-in-oil microemulsions for alkane solvents, is more difficult for carbon dioxide because the properties of carbon dioxide are much different from those of water or nonpolar organic solvents [10]. Carbon dioxide has no dipole moment and weaker van der Waals forces than hydrocarbon solvents. It is possible, however, to form dispersions of either hydrophilic or lipophilic phases in a carbon dioxide continuous phase. Organic-in-carbon dioxide dispersions may be stabilized using surfactants like fluorinated compounds, which are carbon dioxide-philic.

\subsubsection{Solubility in Supercritical Fluids}

According to the ideal gas law, solubility $(\gamma)$ is the ratio of vapor pressure $\left(p_{\mathrm{v}}\right)$ to total pressure $\left(p_{\mathrm{t}}\right)$ in an SCF; however, the behavior is nonideal and the solubility raises several orders of magnitude. The reason for this increase in the solubility is due to the increase in the density of the SCF. Increase in solubility is defined by the enhancement factor $(E)$ that is merely the ratio of actual solubility to the solubility predicted by the ideal gas law.

$$
(E)=\gamma \frac{p_{\mathrm{t}}}{p_{\mathrm{v}}}
$$

Solubility for a given solute also depends on the SCF itself. Different supercritical fluids have different solubilizing efficiencies. This difference arises due to various intermolecular interactions occurring between the solvent and the solute, which can be explained by the solvent polarity. Here the "like dissolves like" rule applies. Thus, a polar solvent is expected to dissolve a polar solute more efficiently than a nonpolar one. Similarly, the structure similarity of both the solvent and solute plays role in the solubility efficiency.

As an example from typical basic applications, as expressed in previous parts, the solubility of naphthalene in $\mathrm{SCCO}_{2}$ is shown in Figure 3.6. As one would expect, at low pressure its solubility is essentially nil. As the pressure of the gas is increased to above the critical pressure of carbon dioxide (which is $73 \mathrm{~atm}$ ), the solubility rises, and for many compounds including naphthalene, the rise is often quite dramatic. For example, at $200 \mathrm{~atm}$ and $45^{\circ} \mathrm{C}$, the solubility is $7 \%$. The solubility behavior shown in Figure 3.6 is the basis of almost all the supercritical fluid extraction/separation processes in operation throughout the world: soluble components are extracted from a substrate by a highpressure gas, and the extracted components that have been dissolved in the gas are precipitated from the gas when the pressure is reduced, for example, across a pressure reduction valve.

The solubility of components in SCFs can be further enhanced by the addition of a substance referred to as an entrainer, or cosolvent. As volatility of this additional component is usually intermediate to that of the SCF and the solute, the addition of cosolvent provides a further dimension to the range of solvent properties in a given system by influencing the chemical nature of the fluid. Cosolvents also provide a mechanism by which the extraction selectivity can be manipulated. The commercial potential of a commercial application of SCF technology can be significantly improved through the use of cosolvents. A factor that must be taken into consideration when using cosolvents, however, is that even the presence of small amounts of an additional component to a primary SCF can change the critical properties of the resulting mixture considerably. 
Starting in the 1960s, many research groups, primarily in Europe, and then later in the United States, examined SCFs for developing advanced extraction processes. European researchers emphasized extraction from botanical substrates, for example, spices, herbs, coffee, tea, and so on, using predominantly $\mathrm{SCCO}_{2}$, and by the 1980s there were several large SCF extraction processes in operation in Germany, the United Kingdom, and the United States, for decaffeinating coffee and tea and extracting flavors and essential oils from hops, spices, and herbs. As an example of size, a coffee decaffeination plant in Bremen processes more than $60,000,000 \mathrm{~kg} /$ year.

The major motivation for developing these SCF processes was the elimination of residual solvents in the products, especially methylene chloride, which had been previously used to decaffeinate coffee. Solvent residues in pharmaceutical and food products were becoming the focus of regulatory attention in the 1970s, and today increasing regulatory attention is being directed to solvent residues. Besides the elimination of solvent residues, there are also other advantages that accrue from employing supercritical fluids in coffee, spices, and herbs, i.e., enhanced flavor and aroma characteristics that cannot be obtained by the traditional organic solvent extraction processes.

Besides the enhanced flavor characteristics and frequently higher yields associated with SFE, some other technical and economic advantages reside in the use of carbon dioxide for the extraction of hop flavors. Organic solvents such as methylene chloride or hexane have previously been the solvents used for the extraction of hops [8]. To obtain the concentrated flavors, it was necessary to distill off the organic solvents, and some of the top note aromas are lost during this step. Carbon dioxide produces a superior product because the top notes are not distilled off, and, as mentioned above, the issue of solvent residues, which is a constant spectre, is eliminated by the use of carbon dioxide.

\subsubsection{Extraction with Supercritical Fluids}

The SFE has been applied only recently to sample preparation on an analytical scale. With advances in process, equipment, and product design, and realization of the potentially profitable opportunities in the production of high value-added products, industries are becoming more and more interested in supercritical fluid technology [11]. The extraction is carried out in high-pressure equipment in a batch or continuous manner as depicted in Figures 3.7 and 3.8, respectively. In both cases, the supercritical solvent is put in contact with the material from which a desirable product is to be separated.

Supercritical extraction has been applied to a large number of solid matrices. The desired product can be either the extract or the extracted solid itself. This technique resembles Soxhlet extraction except that the solvent used is a supercritical fluid, a substance above its critical

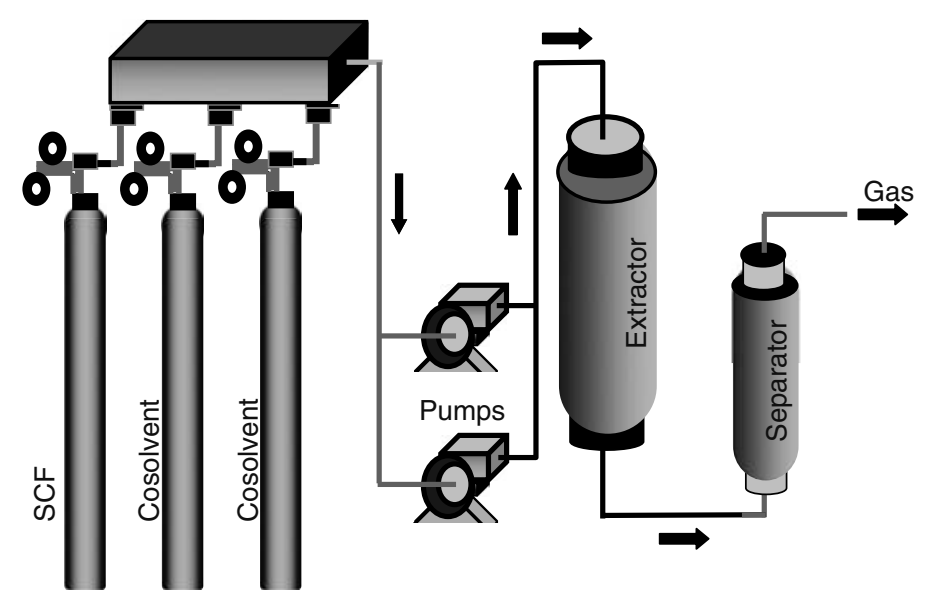

FIGURE 3.7 Schematic diagram of an SCF batch extraction. 


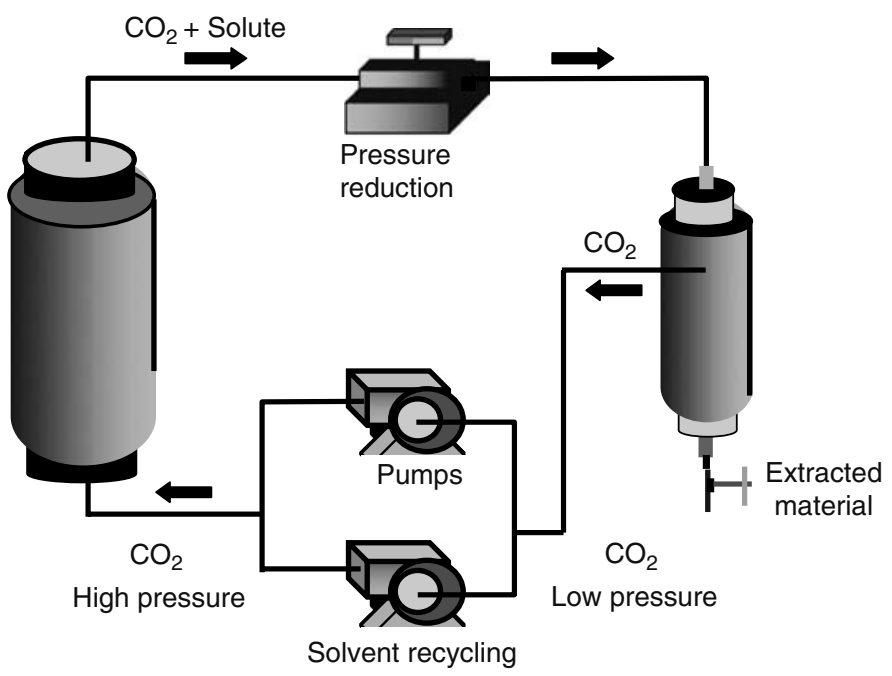

FIGURE 3.8 Schematic diagram of an SCF continuous extraction.

temperature and pressure. This fluid provides a broad range of useful properties [12]. The advantage of using supercritical fluids in extraction is the ease of separation of the extracted solute from the supercritical fluid solvent by simple expansion. In addition, supercritical fluids have liquid-like densities but superior mass transfer characteristics compared to liquid solvents due to their high diffusion and very low surface tension that enables easy penetration into the porous structure of the solid matrix to release the solute. SFE is a relatively new technique in the field of analytical chemistry, having evolved in the last decade as an alternative method of preparing samples before analysis. SFE offers to the analysts many advantages that are not inherent in other sample preparation techniques, such as distillation, extraction with liquid solvents, or low resolution liquid chromatography. The most unique property of supercritical fluids for extraction purposes is the ability to adjust their solubilizing power primarily via mechanical compression (and additionally via temperature), thereby providing the possibility of using one supercritical fluid to extract a host of analytes of varying polarity and molecular size [13]. In addition, solute-fluid binary diffusion coefficients are much greater in supercritical fluid media than in liquid-liquid systems, thereby facilitating fast extraction from a variety of sample matrices.

Furthermore, several legislative protocols (such as the EPA Pollution Prevention Act in the USA) have focused on advocating a reduction in the use of organic solvents, which could be harmful to the environment. The proper choice of supercritical fluid can also provide specific advantages when applied in sample workup before analysis. In addition, the extraction rates are enhanced and less degradation of solutes occurs. Several studies have shown that SFE is a replacement method for traditional gravimetric techniques. In addition, carbon dioxide, which is the most adopted supercritical fluid, has low cost, is a nonflammable compound and devoid of oxygen, thus protecting lipid samples against any oxidative degradation. For example, the low critical temperature of supercritical $\mathrm{CO}_{2}$ makes it an excellent candidate for extracting thermally labile compounds under conditions slightly above room temperature. In addition, $\mathrm{CO}_{2}$ provides an extraction environment free from molecular oxygen, thereby limiting potential oxidation of the extracted solutes. Supercritical $\mathrm{CO}_{2}$, unlike many liquid extraction solvents, is a nontoxic extraction medium; hence, its use in a laboratory environment can eliminate the cost and problems associated with solvent disposal as well as longterm exposure of laboratory personnel to potential toxic vapors.

In practice, SFE can provide appreciable savings in time and cost associated with sample preparation. In general, large polar compounds exhibit almost no solubility in supercritical $\mathrm{CO}_{2}$, 
making it an excellent extraction medium for the separation of nonpolar to moderately polar solutes from such matrices as inorganic solids. However, the solubility of polar analytes can be enhanced in many supercritical fluids by the addition of cosolvents, or modifiers, at low levels to the dense gaseous-phase. By far, the most widely used extraction fluid has been supercritical $\mathrm{CO}_{2}$; however, the extractability of polar solutes can be improved by using a more polar supercritical fluid. Taking $\mathrm{CO}_{2}$ into consideration, the problem with most of the fluids besides $\mathrm{CO}_{2}$ is that these are either difficult to handle or obtain in a pure form.

The following are the advantages of SFEs:

1. Supercritical fluids have a higher diffusion coefficient and lower viscosity than liquids.

2. Absence of surface tension allows for their rapid penetration into the pores of heterogeneous matrices, which helps enhance extraction efficiencies.

3. Selectivity during extraction may be manipulated by varying the conditions of temperature and pressure affecting the solubility of the various components in the supercritical fluid.

4. Supercritical fluid extraction does not leave a chemical residue.

5. Supercritical fluid extractions can use carbon dioxide gas, which can be recycled and used again as part of the unit operation.

Supercritical carbon dioxide has been researched for potential applications in many different fields including food/agriculture, analytical/supercritical fluid chromatography, and the petrochemical/ chemical industries.

Many of the supercritical fluids would not be suitable for practical extractions due to their unfavorable physical properties, costs, or reactivities. For example, ethylene, which exhibits a subambient critical temperature, has been widely investigated in the laboratory as an extractant. However, its flammability limits its application in many analytical problems. Conversely, most polar fluids have high critical temperatures, which can prove destructive to both the analyte and the extraction system. Other fluids, like fluoroform, are unique in their ability to solubilize basic solutes through intermolecular hydrogen bonding in the supercritical fluid state 4 , but the exorbitant cost of the fluid limits its use for SFE. It is useful to compare the physical properties exhibited by $\mathrm{CO}_{2}$, under SFE conditions to those associated with liquid solvents under ambient conditions to gain a better understanding of the advantages, which are attendant to conducting extractions in the supercritical fluid state. Table 3.2 compares the physical properties of $\mathrm{CO}_{2}$ under typical SFE conditions with parameters calculated for three liquid solvents: $n$-hexane, methylene chloride, and methanol at ambient conditions. The density of $\mathrm{CO}_{2}$ at the above conditions is greater than the corresponding value for $n$-hexane, but lower than the densities exhibited by methanol or methylene chloride. Although density is only an approximate measure of

\section{TABLE 3.2}

\section{Comparison of Physical Properties of Supercritical $\mathrm{CO}_{2}$ with Liquid Solvents at $25^{\circ} \mathrm{C}$}

\begin{tabular}{lllcl} 
& $\mathrm{CO}_{2}^{\mathrm{a}}$ & $\boldsymbol{n}$-Hexane & Methylene Chloride & Methanol \\
Density $(\mathrm{g} / \mathrm{mL})$ & 0.746 & 0.660 & 1.326 & 0.791 \\
Kinematic viscosity $\left(\mathrm{m}^{2} / \mathrm{s} \times 10^{7}\right)$ & 1.00 & 4.45 & 3.09 & 6.91 \\
Diffusivity of benzoic acid $\left(\mathrm{m}^{2} / \mathrm{s} \times 10^{9}\right)$ & 6.0 & 4.0 & 2.9 & 1.8 \\
$\left(\frac{P_{\text {v.sat }} \text { solvent }}{P_{\text {v.sat }} \text { solute }}\right)^{\mathrm{b}}$ & $1.4 \times 10^{5}$ & $4.2 \times 10^{2}$ & $1.2 \times 10^{3}$ & $3.6 \times 10^{2}$ \\
a At 200 atm and $55^{\circ} \mathrm{C}$. & & & & \\
b Solute is phenol at $25^{\circ} \mathrm{C}$. & & & \\
& & & & \\
\hline
\end{tabular}


intermolecular attraction, the value for $\mathrm{CO}_{2}$ suggests that near liquid-like densities can be achieved for this gas in its supercritical fluid state. Likewise, kinetic-based properties such as viscosity and solute diffusivity, for $\mathrm{CO}_{2}$, have values that are more typical of gases than those of the liquid state. These gas-like transport parameters contribute to improved rates of mass transfer for solutes in supercritical fluid media, resulting in faster extraction. The ratio of the saturated vapor pressures of the extraction solvents to that exhibited by a typical solute, phenol, at $25^{\circ} \mathrm{C}$ is also tabulated in Table 3.2.

\subsection{SUPERCRITICAL FLUID EXTRACTION MECHANISM}

Liquid-solid extraction techniques are widely used for isolation of analytes from a solid matrix. One such technique, solid extraction, involves repeated solvent distillation through a solid sample to remove the analyte of interest. This technique is often used for extracting additives from polymers and organics from soils. Not only does Soxhlet extraction requires the use of an organic solvent that will eventually require disposal but also the technique is sometimes very slow. A relatively new extraction technique for isolation of analytes from solid samples is SFE. It has been considered in some studies that SFE collects great attention and interest because of providing short sample preparation time and being better than the conventional extraction techniques [14].

Extraction of soluble species (solutes) from solid matrices takes place through four different mechanisms:

- If there are no interactions between the solute and the solid phase, the process is simple dissolution of the solute in a suitable solvent that does not dissolve the solid matrix.

- If there are interactions between the solid and the solute, then the extraction process is termed as desorption and the adsorption isotherm of the solute on the solid in presence of the solvent determines the equilibrium. Most solids extraction processes, such as activated carbon regeneration, fall in this category.

- Third mechanism is swelling of the solid phase by the solvent accompanied by extraction of the entrapped solute through the first two mechanisms, such as extraction of pigments or residual solvents from polymeric matrices.

- Fourth mechanism is reactive extraction where the insoluble solute reacts with the solvent and the reaction products are soluble hence extractable, such as extraction of lignin from cellulose. Extraction is always followed by another separation process where the extracted solute is separated from the solvent.

Another important aspect in supercritical extraction relates to solvent/solute interactions. Normally, the interactions between the solid and the solute determine the ease of extraction, i.e., the strength of the adsorption isotherm is determined by interactions between the adsorbent and the adsorbate. However, when supercritical fluids are used, interactions between the solvent and the solute affect the adsorption characteristics due to large negative partial molar volumes and partial molar enthalpies in supercritical fluids.

The thermodynamic parameters that govern the extraction are found to be temperature, pressure, the adsorption equilibrium constant, and the solubility of the organic in supercritical fluid [9]. Similar to the retrograde behavior of solubility in supercritical fluids, the adsorption equilibrium constants can either decrease or increase for an increase in temperature at isobaric conditions. This is primarily due to the large negative partial molar properties of the supercritical fluids. In addition to the above factors, the rate parameters like the external mass transfer resistances, the axial dispersion in the fluid phase, and the effective diffusion of the organics in the pores also play a crucial role in the desorption process. A thorough understanding of these governing parameters is important in the modeling of SFE process and in the design, development, and future scale-up of the process. 


\subsection{SUPERCRITICAL FLUID EXTRACTION THEORY}

In an effort to understand the parameters influencing SFE on the analytical scale, many researchers have studied the thermodynamics of solubility in SCFs and extended this knowledge to supercritical fluid extraction (SCFE) models. There has been a wealth of information relating to SCFE in the chemical engineering and physical chemistry literature. Many simple and relatively fast liquid solvent extraction techniques exist, and when such extractions can be conveniently performed, are quantitative, and do not require concentration for the determination of target analytes, SCFE has few apparent advantages other than the reducing solvent usage.

The density of SCF and the Hildebrand solubility parameter $(\delta)$ increases with increasing pressure. The following semiempirical relationship is defined relating the Hildebrand solubility parameter to the density of an SCF:

$$
\delta=0.47 P_{\mathrm{c}}^{1 / 2} \rho
$$

Here, $\rho$ is the density of the SCF, which is related to pressure and temperature. The equation is used to calculate the Hildebrand solubility parameter for various SCFs.

The same procedure is used to calculate the Hildebrand solubility parameter for binary fluids. However, the relationship between the solvent strength of a mixed SCF and its density is no longer valid for binary fluids that contain a polar component (modifier). Hildebrand solubility parameters are fairly good predictors of extraction efficiency if the sample matrix has no strong adsorption sites. However, if polar analytes are adsorbed onto a polar sample matrix with relatively strong adsorption sites, small amounts of polar modifier will greatly enhance their desorption. Recovery is vastly improved as compared to the use of pure, unmodified SCF.

The effect of temperature on the solid solubility is different at pressures in the critical range or when the system pressure exceeds the critical value by a factor of two or more. Near the system critical pressure, the fluid density is very sensitive to temperature. Therefore, a moderate increase in temperature leads to a large decrease in fluid density with the consequent reduction in solid solubility. At pressures well above the SCF critical pressure, the solute solubility isotherms exhibit a maximum. It was shown that the maximum is achieved when the partial molar volume of the solute in the fluid phase is equal to the solute solid molar volume [15]. A quantitative correlation and prediction of the solubility of a pure solid in a supercritical gas are possible if the fugacity coefficient of the solid in the gas phase can be obtained from an equation of state.

\subsection{EXPERIMENTAL CONSIDERATIONS}

The extraction concept is not difficult and complex to perform. The process is simple, with the major process parameters being temperature, pressure, and flow rate of the supercritical fluid. Figure 3.9 presents a basic flow diagram for SFE. Mainly, to obtain the desired pressure value, a pump is used and the extraction fluid is supplied to the extraction cell that is placed in an electric oven. The temperature is kept in a value above the critical temperature of SCF. In this case, the supercritical solvent is put into contact with the material from which a desirable product is to be separated. During SFE, the supercritical solvent, saturated with the extracted compound, is expanded to the atmospheric conditions and the solubilized product is recovered in the separation vessel permitting the recycle of the supercritical solvent for further use [16]. A schematic diagram for a typical SFE system is illustrated in Figure 3.10.

The system is basically formed of a liquid $\mathrm{CO}_{2}$ cylinder, a pump which is preferred mostly to be of syringe type to keep the pressure at an adjusted value [17]. The pressure is kept above the critical pressure and the temperature of the extraction vessel is controlled in supercritical conditions. The extraction process takes place in extraction vessel. After extraction, SCF is passed at a lower pressure and goes through the receiver. $\mathrm{CO}_{2}$, containing droplets and dissolved substances, leaving the receiver is passed through a demister and a carbon scrubber before being recycled to the 


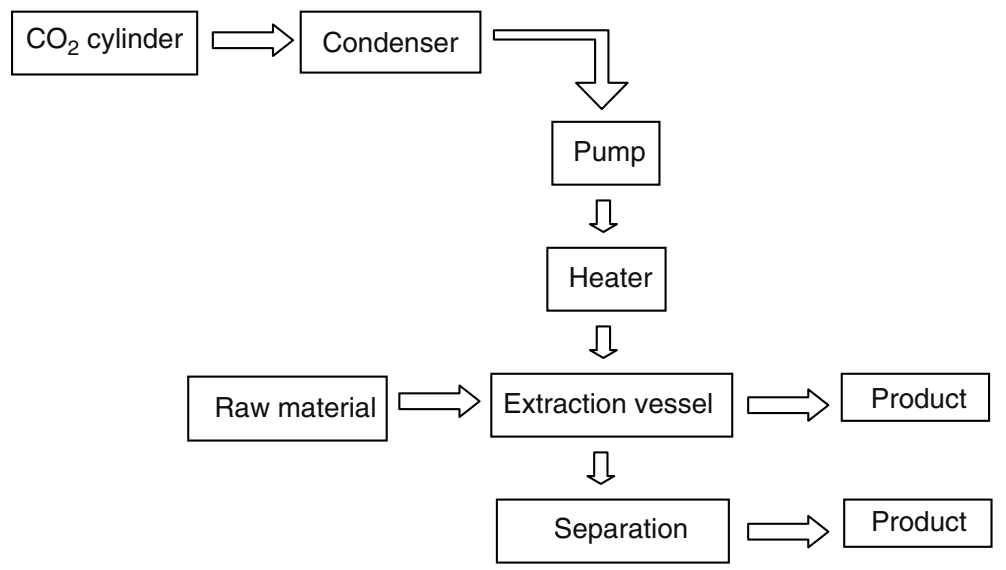

FIGURE 3.9 Flow diagram of an SFE system.

liquefaction unit [9]. As a following step, separation of desired component from the stream by SFE can be achieved in different ways. One way is to precipitate the solute from the solvent by reduction of the solvent density that is done by reduction in pressure, increase in temperature, or mixing the extract with atmospheric gases like Ar or $\mathrm{N}_{2}$. Sometimes, the product is recovered from the extract by washing it with a suitable solvent.

One should consider the importance of some parameters such as density, diffusivity, critical temperature, critical pressure, etc. so that the SCF can be chosen carefully to carry out the extraction process efficiently in which the extraction pressure and temperature are kept constant at desired values for desired extraction time of the materials at prepared sample sizes.

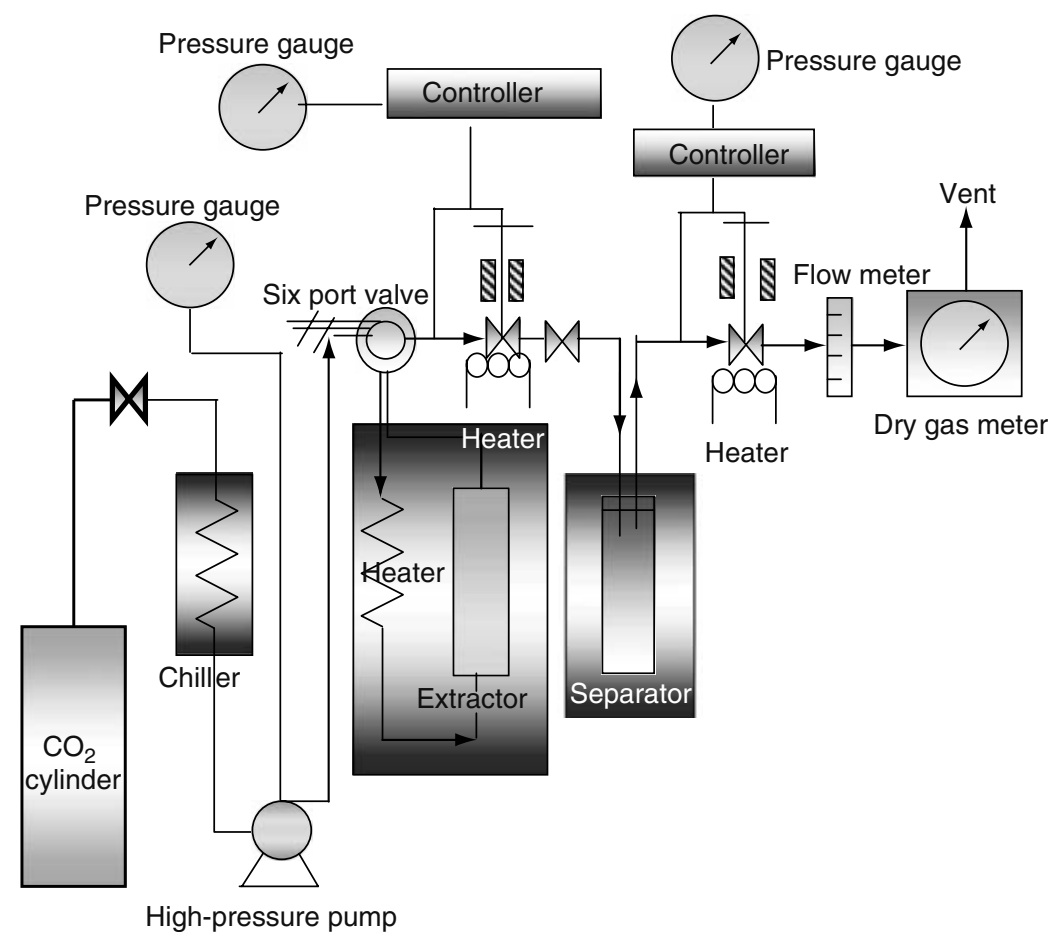

FIGURE 3.10 SFE apparatus. 